\title{
A FOURTH ORDER CURVATURE FLOW ON A CR 3-MANIFOLD
}

\author{
${ }^{*}$ SHU-CHENG CHANG ${ }^{1},{ }^{*}$ JIH-HSIN CHENG ${ }^{2}$, AND HUNG-LIN CHIU ${ }^{3}$
}

\begin{abstract}
Let $\left(\mathbf{M}^{3}, J, \theta_{0}\right)$ be a closed pseudohermitian 3-manifold. Suppose the associated torsion vanishes and the associated $Q$-curvature has no kernel part with respect to the associated Paneitz operator. On such a background pseudohermitian 3-manifold, we study the change of the contact form according to a certain version of normalized $Q$-curvature flow. This is a fourth order evolution equation. We prove that the solution exists for all time and converges smoothly to a contact form of zero $Q$-curvature. We also consider other background conditions and obtain a priori bounds up to high orders for the solution.
\end{abstract}

\section{Introduction}

Let $(M, J, \theta)$ be a closed (i.e., compact with no boundary) pseudohermitian 3manifold (see Appendix A for basic notions in pseudohermitian geometry). In the papers $[\mathrm{FH}],[\mathrm{GG},[\mathrm{H}]$, and $[\mathrm{GL}$, the $(C R)$ Paneitz operator $P$ (acting on a smooth real function $\lambda)$ with respect to $(J, \theta)$ is defined by

$$
P \lambda=\Delta_{b}^{2} \lambda+T^{2} \lambda+4 \operatorname{Im}\left(A_{\overline{11}} \lambda_{11}+A_{\overline{11}, 1} \lambda_{1}\right),
$$

and the so-called $Q$-curvature is defined by

$$
Q=-\frac{2}{3}\left(\Delta_{b} W+2 \operatorname{Im} A_{11, \overline{11}}\right)
$$

where $\Delta_{b}, T, W$, and $A_{\overline{11}}$ denote the sub-Laplacian, the characteristic vector field, the Tanaka-Webster (scalar) curvature, and the torsion with respect to $(J, \theta)$, respectively. Moreover, for a contact form change $\theta=e^{2 \lambda} \theta_{0}$, we have the following transformation laws:

$$
P=e^{-4 \lambda} P_{0}
$$

1991 Mathematics Subject Classification. Primary 32V20; Secondary 53C44.

Key words and phrases. CR Manifold, Tanaka-Webster Curvature, Torsion, Moser inequality, $Q$-curvature flow, Paneitz operator, Sub-Laplacian, Kohn Laplacian.

* Research supported in part by the NSC of Taiwan. 
and

$$
Q=e^{-4 \lambda}\left(Q_{0}+2 P_{0} \lambda\right)
$$

where $P_{0}$ and $Q_{0}$ denote the $(C R)$ Paneitz operator and the $Q$-curvature with respect to $\left(J, \theta_{0}\right)$, respectively. Note that

$$
\int_{M} Q d \mu=0
$$

Here the volume form $d \mu=\theta \wedge d \theta$.

Then we ask if we can always choose $\theta$ so that $Q$ vanishes pointwise. The related problems for the $Q$-curvature on a Riemannian manifold are also addressed and studied in [B], [C1], and [CW] by using the method of the $Q$-curvature flow. In this paper we study a similar evolution equation and apply it to the problem addressed above on a $C R$ 3-manifold.

We consider the functional $\mathcal{E}$ on a closed $C R$ 3-manifold $(M, J)$ with a given contact class $\left[\theta_{0}\right]$ (which consists of all contact forms annihilating the underlying contact bundle):

$$
\mathcal{E}(\theta)=\int_{M} P_{0} \lambda \cdot \lambda d \mu_{0}+\int_{M} Q_{0} \lambda d \mu_{0}
$$

for $\theta=e^{2 \lambda} \theta_{0}$, where $d \mu_{0}=\theta_{0} \wedge d \theta_{0}$. Then, for minimizing $\mathcal{E}(\theta)$ in $\left[\theta_{0}\right]$, it is natural to consider the following fourth order ( $Q$-curvature) flow on a closed $C R 3$-manifold $(M, J)$ :

$$
\left\{\begin{array}{l}
\frac{\partial \lambda}{\partial t}=-\left(Q_{0}+2 P_{0} \lambda\right)+r=-e^{4 \lambda} Q+r \\
\theta=e^{2 \lambda} \theta_{0} ; \lambda(p, 0)=\lambda_{0}(p) \\
\int_{M} e^{4 \lambda_{0}} d \mu_{0}=\int_{M} d \mu_{0}
\end{array}\right.
$$

where

$$
r=\frac{\int_{M}\left(Q_{0}+2 P_{0} \lambda\right) d \mu}{\int_{M} d \mu}=\frac{\int_{M} e^{4 \lambda} Q d \mu}{\int_{M} d \mu}
$$

and $\lambda_{0}$ is an initial real $C^{\infty}$ smooth function. Note that $d \mu=e^{4 \lambda} d \mu_{0}$ and the volume $V=\int_{M} d \mu$ is kept invariant under the flow (1.6). Indeed (1.6) is the (volume normalized) negative gradient flow of $\mathcal{E}(\theta)$. That is, by using (1.4) and (1.3), one can 
check that

$$
\begin{aligned}
& \frac{d}{d t} \mathcal{E}(\theta) \\
& =2 \int_{M} P_{0} \lambda \cdot \frac{\partial \lambda}{\partial t} d \mu_{0}+\int_{M} Q_{0} \frac{\partial \lambda}{\partial t} d \mu_{0} \\
& =-2 \int_{M}\left(Q_{0}+2 P_{0} \lambda\right) P_{0} \lambda d \mu_{0}-\int_{M} Q_{0}\left(Q_{0}+2 P_{0} \lambda\right) d \mu_{0} \\
& =-\int_{M}\left(Q_{0}+2 P_{0} \lambda\right)^{2} d \mu_{0} \\
& =-\int_{M} e^{4 \lambda} Q^{2} d \mu .
\end{aligned}
$$

under the flow (1.6). We will often denote a $C R$ 3-manifold by $\left(M, J,\left[\theta_{0}\right]\right)$ with its contact class (or equivalently its underlying contact bundle) indicated. Let $A_{11}$ denote the torsion with respect to $\left(J, \theta_{0}\right)$. For the long time solution to the flow (1.6), we have the following result.

Theorem 1.1. Let $\left(M, J,\left[\theta_{0}\right]\right)$ be a closed $C R$ 3-manifold with $\stackrel{0}{A_{11}}=0$. Then the solution of (1.6) exists on $M \times[0, \infty)$.

There are many torsion free examples. On the other hand, this condition implies strong topological obstruction (see the Appendix in $[\mathrm{CH}]$ ). We hope this condition can be weakened in the future study.

To motivate the definitions of some analytic conditions, let us first examine the standard $C R$ 3-sphere $\mathbf{S}^{3}=\left\{\left(z_{0}, z_{1}\right) \mid \sum_{j=0}^{1} z_{j} \bar{z}_{j}=1\right\} \subset \mathbf{C}^{2}$ with the induced $C R$ structure $J$ from $\mathbf{C}^{2}$ and the contact form $\theta_{0}=\left.\frac{i(\bar{\partial} u-\partial u)}{2}\right|_{\mathbf{S}^{3}}$, where $u=\left(\sum_{j=0}^{1} z_{j} \bar{z}_{j}\right)-1$ is a defining function. With respect to $\left(J, \theta_{0}\right), \mathbf{S}^{3}$ is torsion free and $Q_{0}=0$. On the other hand, if $f_{p, q}$ is a bigraded spherical harmonic of type $(p, q)$ on $\mathbf{C}^{2}$ (i.e. a harmonic polynomial which is a linear combination of terms of the form $z^{\rho} \bar{z}^{\gamma}$ ), then we have $($ Chi $])\left(\Delta_{0}\right.$ denotes the sub-Laplacian with respect to $\left.\left(J, \theta_{0}\right)\right)$

$$
\Delta_{0} f_{p, q}=-(2 p q+p+q) \cdot f_{p, q}
$$

and

$$
P_{0} f_{p, q}=4 p q(p+1)(q+1) \cdot f_{p, q}
$$

Definition 1.1. On a closed pseudohermitian manifold $(M, J, \theta)$, we call the Paneitz operator $P$ with respect to $(J, \theta)$ essentially positive if there exists a constant $\Upsilon>0$ 
such that

$$
\int_{M} P \varphi \cdot \varphi d \mu \geq \Upsilon \int_{M} \varphi^{2} d \mu
$$

for all real $C^{\infty}$ smooth functions $\varphi \perp \operatorname{Ker}(P)$ (i.e. perpendicular to the kernel of $P$ in the $L^{2}$ norm with respect to the volume form $\left.d \mu=\theta \wedge d \theta\right)$.

Remark 1.1. We note that $P$ being essentially positive is a $C R$ invariant property by (1.2), i.e., it is independent of the choice of contact form. For $M$ being the boundary of a bounded strictly pseudoconvex domain in $C^{2}, P$ appears in the transformation law of the first invariant in the logarithmic term of Fefferman's asymptotic expansion of the Szegö kernel $([\mathrm{H}])$. Also $P$ appears as the compatibility operator for the degenerate Laplacian in the paper GL. On the other hand, the kernel of $P$ is infinite dimensional, containing all $C R$-pluriharmonic functions (see Section 5). So even for the short time solution to (1.6), we need to treat the kernel part separately. The condition that $P$ is essentially positive was also used to study the problem of the first eigenvalue of the sub-Laplacian (see [Chi]).

Since the restrictions of bigraded spherical harmonics to $\mathbf{S}^{3}$ span a dense subspace of $L^{2}\left(\mathbf{S}^{3}\right)$ (see Proposition 12.3.3 in [CS]), we conclude that the Paneitz operator $P_{0}$ of $\left(\mathbf{S}^{3}, J, \theta_{0}\right)$ is essentially positive by (1.9). Combining (1.8) and (1.9), we get

$$
\left(2 P_{0}-\Delta_{0}^{2}\right) f_{p, q}=\lambda(p, q) f_{p, q}
$$

where $\lambda(p, q)=p^{2}(4 q-1)+q^{2}(4 p-1)+6 p q+4 p^{2} q^{2}$. It is clear that if $P_{0} f_{p, q} \neq 0$, i.e., $p q \neq 0$, then $\lambda(p, q)>0$. This means that the operator $2 P_{0}-\Delta_{0}^{2}$ is positive on the orthogonal complement of the Kernel of $P_{0}$. Therefore if $\varphi$ is a real $C^{\infty}$ smooth function such that $\varphi \perp \operatorname{Ker}\left(P_{0}\right)$, then

$$
2 \int_{\mathbf{S}^{3}} P_{0} \varphi \cdot \varphi d \mu_{0} \geq \int_{\mathbf{S}^{3}} \Delta_{0}^{2} \varphi \cdot \varphi d \mu_{0}=\int_{\mathbf{S}^{3}}\left(\Delta_{0} \varphi\right)^{2} d \mu_{0}
$$

Let $\stackrel{0}{\nabla}$ and $\stackrel{0}{\nabla^{2}}$ denote the sub-gradient and the sub-Hessian with respect to $\left(J, \theta_{0}\right)$, respectively. Now based on the Bochner formula, we have (Lemma 3.2 in Section 3; 
see Chi also)

$$
\begin{aligned}
\int_{M}\left|\stackrel{0}{\nabla}^{2} \varphi\right|^{2} d \mu_{0}= & 3 \int_{M}\left(\Delta_{0} \varphi\right)^{2} d \mu_{0}-2 \int_{M} P_{0} \varphi \cdot \varphi d \mu_{0} \\
& -\int_{M} \stackrel{0}{W}|\stackrel{0}{\nabla} \varphi|^{2} d \mu_{0}-6 \operatorname{Im} \int_{M} \stackrel{0}{A}_{\overline{11}} \varphi_{1} \varphi_{1} d \mu_{0}
\end{aligned}
$$

where $\stackrel{0}{W}$ and $\stackrel{0}{A}_{\overline{11}}$ denote the Tanaka-Webster curvature and the torsion with respect to $\left(J, \theta_{0}\right)$, respectively.

Observe that $\stackrel{0}{W}$ is a positive constant and $\stackrel{0}{A} \overline{11}=0$ for $\left(\mathbf{S}^{3}, J, \theta_{0}\right)$. It follows from (1.11) and (1.12) that

$$
\int_{\mathbf{S}^{3}}\left|\nabla^{2} \varphi\right|^{2} d \mu_{0} \leq 2 \int_{\mathbf{S}^{3}}\left(\Delta_{0} \varphi\right)^{2} d \mu_{0}
$$

for $\varphi \perp \operatorname{Ker}\left(P_{0}\right)$.

Inspired by the inequality (1.13), we make the following definition.

Definition 1.2. We say that the condition $(*)$ is satisfied on a closed CR 3-manifold $\left(M, J,\left[\theta_{0}\right]\right)$ if there exist constants $0 \leq \varepsilon_{0}<1$ and $C\left(\varepsilon_{0}\right) \geq 0$ such that

$$
\int_{M}\left|\nabla^{2} \varphi\right|^{2} d \mu_{0} \leq\left(2+\varepsilon_{0}\right) \int_{M}\left(\Delta_{0} \varphi\right)^{2} d \mu_{0}+C\left(\varepsilon_{0}\right) \int_{M} \varphi^{2} d \mu_{0}
$$

for all real $C^{\infty}$ smooth functions $\varphi \perp \operatorname{Ker}\left(P_{0}\right)$.

Theorem 1.2. Let $\left(M, J,\left[\theta_{0}\right]\right)$ be a closed $C R$ 3-manifold. Suppose that $P_{0}$ is essentially positive and the condition $(*)$ holds. Then the solution $\lambda$ of (1.6) satisfies an a priori $S^{2,2}$ estimate:

$$
\|\lambda\|_{S^{2,2}} \leq C(T)
$$

for $t \in[0, T)$, where $C(T)$ is a constant depending on T. Moreover, if in addition $\left(Q_{0}\right)_{\mathrm{ker}}=0$, then the bound $C(T)$ can be replaced by a constant $C$ independent of the time.

In the paper $[\mathrm{B}$, the (essential) positivity of the Riemannian Paneitz operator is also needed for a similar result on the $Q$-curvature flow on a closed conformal 4manifold $\left(M,\left[g_{0}\right]\right)$ with a given conformal class $\left[g_{0}\right]$. However, in the Riemannian case, the condition analogous to the condition $(*)$ holds always. We wonder to what extent the condition $(*)$ is valid for a closed pseudohermitian manifold. 
In Section 5, we show that if the torsion $\stackrel{0}{A} A_{11}$ of $\left(M, J, \theta_{0}\right)$ is zero, then the $C R$ Paneitz operator $P_{0}$ is essentially positive. In that case, the $C R$ Paneitz operator $P_{0}=\square_{b} \overline{\square_{b}}$ and the Kohn Laplacian $\square_{b}$ and $\overline{\square_{b}}$ commute (see Section 5). We can write an $L^{2}$ function $\varphi=\varphi_{\text {ker }}+\varphi^{\perp}$ where $\varphi_{\text {ker }} \in \operatorname{Ker}\left(P_{0}\right)$ and $\varphi^{\perp} \in \operatorname{Ker}\left(P_{0}\right)^{\perp}$. We have the following a priori estimates of higher orders.

Theorem 1.3. Let $\left(M, J,\left[\theta_{0}\right]\right)$ be a closed $C R$ 3-manifold. Suppose that $P_{0}$ is essentially positive and the condition $(*)$ holds. In addition, suppose also that $\Delta_{0}\left(\operatorname{Ker}\left(P_{0}\right)\right)$ $\subset \operatorname{Ker}\left(P_{0}\right)$. Then for any nonnegative integer $k$, the solution $\lambda$ of (1.6) satisfies an a priori $S^{2 k, 2}$ estimate:

$$
\|\lambda\|_{S^{2 k, 2}} \leq C(k, T)
$$

for $t \in[0, T)$, where $C(k, T)$ is a constant depending on $k$ and $T$. Moreover, if in addition $\left(Q_{0}\right)_{\mathrm{ker}}=0$, then the bound $C(k, T)$ can be replaced by a constant $C(k)$ independent of the time.

We remark that in the torsion free case, the condition $\Delta_{0}\left(\operatorname{Ker}\left(P_{0}\right)\right) \subset \operatorname{Ker}\left(P_{0}\right)$ holds true (also $P_{0}$ is essentially positive as mentioned above). We have the following asymptotic convergence of solutions of (1.6) .

Theorem 1.4. Let $\left(M, J,\left[\theta_{0}\right]\right)$ be a closed CR 3-manifold with $\left(Q_{0}\right)_{\mathrm{ker}}=0$. Suppose that $\stackrel{0}{A}_{11}=0$. Then the solution of (1.6) exists on $M \times[0, \infty)$ and converges smoothly to $\lambda_{\infty} \equiv \lambda(\cdot, \infty)$ as $t \rightarrow \infty$. Moreover, the contact form $e^{2 \lambda_{\infty}} \theta_{0}$ has zero $Q$-curvature.

Remark 1.2. 1. Let $W_{0}$ denote the Tanaka-Webster (scalar) curvature with respect to $\left(J, \theta_{0}\right)$. If $\stackrel{0}{A} A_{11}=0$, then $P_{0}$ commutes with $\Delta_{0}$ and hence there holds

$$
\left(Q_{0}\right)_{\mathrm{ker}}=-\frac{2}{3}\left(\Delta_{0} W_{0}\right)_{\mathrm{ker}}=-\frac{2}{3} \Delta_{0}\left(W_{0}\right)_{\mathrm{ker}}
$$

by (1.1). It follows that

$$
\left(Q_{0}\right)_{\mathrm{ker}}=0 \Longleftrightarrow\left(W_{0}\right)_{\mathrm{ker}} \text { is a constant }
$$

2. On the standard $C R$ 3-sphere $\left(\mathbf{S}^{3}, J,\left[\theta_{0}\right]\right)$, we have

(i) $\stackrel{0}{A_{11}}=0$ and $Q_{0}=0$,

(ii) the condition $(*)$ holds with $\varepsilon_{0}=0$ and $C\left(\varepsilon_{0}\right)=0$. 
As a consequence of Theorem 1.4, we have

Corollary 1.5. Let $\left(\mathbf{S}^{3}, J,\left[\theta_{0}\right]\right)$ be the standard $C R$ 3-sphere. Then the solution of (1.6) exists on $\mathbf{S}^{3} \times[0, \infty)$ and converges smoothly to $\lambda_{\infty}$ such that $e^{2 \lambda_{\infty}} \theta_{0}$ is a contact form of zero $Q$-curvature.

We recall that $\theta_{0}$ is called an invariant contact form on a $C R 3$-manifold $M$ if it is locally volume- normalized with respect to a closed $(2,0)$-form on $M([\mathrm{FH}]$, [L1], $[\mathrm{Fa}]$. In the paper $[\mathrm{FH}]$, the authors proved that the $Q$-curvature of an invariant contact form vanishes. Indeed if $M$ is a real hypersurface in $\mathbf{C}^{2}$, then $M$ admits an invariant contact form $\theta_{0}$ so that $Q_{0}=0$ on $M$. In general, there is a topological obstruction for the global existence of an invariant contact form $\theta_{0}$ ([L1] $)$. However, on the standard $C R$ 3-sphere, $Q_{0}=0$ if and only if $\theta_{0}$ is an invariant contact form.

About other curvature flows, we notice that it is still an open problem whether we have the long-time existence and convergence for solutions of the $C R$ Yamabe flow on a closed $C R$ 3-manifold ([CC1], [JL]). This is a second order subparabolic equation. On the other hand, the flow (1.6) which we are dealing with is a fourthorder subparabolic (at least under a certain condition) equation. Because there seems to be no suitable maximum principle available for fourth-order subelliptic operators, we need to invoke a priori $L^{2}$ estimates for solutions to (1.6) in place of the pointwise estimates used for second-order (sub)elliptic operators ([B], C], C1], C2], [CW]). In case $M$ is a surface, the $Q$-curvature flow corresponds to the 2-dimensional Calabi flow which is solved completely by P. T. Chruściel $([\mathrm{C}])$ and the first author ([C3], [C4]; see also [CW]).

In order to get the $S^{2,2}$-estimate (see Appendix A for the definition of FollandStein norms $S^{k, p}$ ), we need an additional analytic condition $(*)$ (which holds for the standard pseudohermitian 3-sphere) plus a trick from S. Brendle's work ([B] $)$ for the $Q$-curvature flow on Riemannian 4-manifolds. This is because the pseudohermitian version of a Bochner-type estimate is entirely different from the Riemannian version (see Lemma 3.1).

We briefly describe the methods used in our proofs. In Section 2, in order to have the $S^{k, 2}$-estimates for $\lambda$, we need to derive the analogue of the Moser inequality on pseudohermitian 3-manifolds. In Section 3, based on a pseudohermitian version 
of the Bochner formula, we first derive a key estimate for the equation (1.6) as in Lemma 3.2, which involves the $C R$ Paneitz operator $P_{0}$. We show the subellipticity of $P_{0}$ on $\left(\operatorname{ker} P_{0}\right)^{\perp}$ under the torsion free condition to get the short time solution. For the long-time solution of (1.6) under the same condition (i.e., Theorem 1.1), we obtain the higher order bounds for the solution.

In Section 4, we derive the $S^{2,2}$-estimate and higher-order $S^{2 k, 2}$-estimates for $\lambda$ under the flow (1.6), and hence prove Theorem 1.2 and Theorem 1.3. Then we prove the smooth convergence of solutions of (1.6) (i.e. Theorem 1.4) by a method analogous to that in $[\mathrm{S}]$. In Section 5 , we show the essential positivity of the $C R$ Paneitz operator $P$ on $(M, J, \theta)$ with the zero torsion.

\section{Moser's Inequality on Pseudohermitian 3-manifolds}

In this section, based on [A], [CL], [FS1], and [SC], we derive the analogue of Moser's inequality on pseudohermitian 3-manifolds.

Let $H^{1}=\mathbf{C} \times \mathbf{R}$ be the (3-dimensional) Heisenberg group with coordinates $(z, t)$. For each real number $r \in \mathbf{R}$, there is a dilation naturally associated with $H^{1}$, which is usually denoted as

$$
\delta_{r} u=\delta_{r}(z, t)=\left(r z, r^{2} t\right)
$$

The anisotropic dilation structure on $H^{1}$ introduces a homogeneous norm

$$
|u|=|(z, t)|=\left(|z|^{4}+t^{2}\right)^{1 / 4} .
$$

With this norm, we can define the Heisenberg ball centered at $u=(z, t)$ with radius $R$ by $B(u, R)=\left\{v \in H^{1}:\left|u^{-1} \cdot v\right|<R\right\}$.

In their paper ([CL), William S. Cohn and Guozhen Lu show that for all $\varphi \in$ $C_{0}^{\infty}\left(H^{1}\right)$,

$$
|\varphi(v)| \leq L^{-1} \int_{H^{1}} \frac{\left|\nabla_{b} \varphi(u)\right|}{\left|v^{-1} \cdot u\right|^{3}} d V(u),
$$

where $L=\frac{2 \pi \Gamma(1 / 2) \Gamma(3 / 4)}{\Gamma(1) \Gamma(5 / 4)}$ and $d V(u)=d x \wedge d y \wedge d t, z=x+i y$. Note that we only take the case $\beta=1$ in Theorem 1.2 of ( $[\mathrm{CL})$.

Let $B$ be the unit Heisenberg ball of $H^{1}$, centered at $(0,0)$. Let $\|\cdot\|_{p}$ denote the $L^{p}$ norm with respect to the volume form $d V(u)$. 
Lemma 2.1. For $\varphi \in C_{0}^{\infty}(B)$ and $p \geq 4$, we have

$$
\|\varphi\|_{p} \leq L^{-1}\left\|\nabla_{b} \varphi\right\|_{4} \sup _{v \in B}\left[\int_{B}\left|v^{-1} \cdot u\right|^{-3 k} d V(u)\right]^{\frac{1}{k}}
$$

where $\frac{1}{k}=\frac{1}{p}+\frac{3}{4}$.

Proof. We write

$$
\begin{aligned}
& \left|v^{-1} \cdot u\right|^{-3}\left|\nabla_{b} \varphi(u)\right| \\
= & \left(\left|v^{-1} \cdot u\right|^{-3 k}\left|\nabla_{b} \varphi(u)\right|^{4}\right)^{\frac{1}{p}}\left(\left|v^{-1} \cdot u\right|^{-3 k}\right)^{\frac{3}{4}}\left(\left|\nabla_{b} \varphi(u)\right|^{4}\right)^{\frac{1}{4}-\frac{1}{p}} .
\end{aligned}
$$

Since $\frac{1}{p}+\frac{1}{4}+\left(\frac{1}{4}-\frac{1}{p}\right)=1$, applying Hölder's inequality to (2.1), we have

$$
\begin{aligned}
|\varphi(v)| \leq L^{-1} & \left(\int_{B}\left|v^{-1} \cdot u\right|^{-3 k}\left|\nabla_{b} \varphi(u)\right|^{4} d V(u)\right)^{\frac{1}{p}}\left(\int_{B}\left|v^{-1} \cdot u\right|^{-3 k} d V(u)\right)^{\frac{3}{4}} \\
& \left(\int_{B}\left|\nabla_{b} \varphi(u)\right|^{4} d V(u)\right)^{\frac{1}{4}-\frac{1}{p}} \cdot
\end{aligned}
$$

This implies that

$$
\begin{aligned}
\|\varphi\|_{p} & =\left(\int_{B}|\varphi(v)|^{p} d V(v)\right)^{\frac{1}{p}} \\
& \leq\left(L^{-1}\left\|\nabla_{b} \varphi\right\|_{4}^{1-\frac{4}{p}} \cdot \Lambda^{\frac{3 k}{4}}\right)\left[\int_{B} \int_{B}\left|v^{-1} \cdot u\right|^{-3 k}\left|\nabla_{b} \varphi(u)\right|^{4} d V(u) d V(v)\right]^{\frac{1}{p}} \\
& \leq L^{-1}\left\|\nabla_{b} \varphi\right\|_{4} \cdot \Lambda
\end{aligned}
$$

where $\Lambda=\sup _{v \in B}\left[\int_{B}\left|v^{-1} \cdot u\right|^{-3 k} d V(u)\right]^{\frac{1}{k}}$.

Corollary 2.2. For all $\varphi \in C_{0}^{\infty}(B)$,

$$
\int_{B} e^{\varphi} d V(u) \leq C \exp \left(\varkappa\left\|\nabla_{b} \varphi\right\|_{4}^{4}\right)
$$

where $C$ and $\varkappa$ are two positive constants.

Proof. Let $\Sigma=\partial B$ be the unit Heisenberg sphere and $d A$ be the unique Radon measure on $\Sigma([\mathrm{CL}],[\mathrm{FS} 1])$. We denote $A_{1}=\int_{\Sigma} d A$. Note that there exists a 
number $\delta$ such that $\left|v^{-1} \cdot u\right|<\delta$ for all $u, v \in B$. This means that $B \subset B(v, \delta)$ for all $v \in B$.

By (2.2), we have

$$
\begin{aligned}
\|\varphi\|_{p} & \leq L^{-1}\left\|\nabla_{b} \varphi\right\|_{4} \sup _{v \in B}\left[\int_{B}\left|v^{-1} \cdot u\right|^{-3 k} d V(u)\right]^{\frac{1}{k}} \\
& \leq L^{-1}\left\|\nabla_{b} \varphi\right\|_{4} \sup _{v \in B}\left[\int_{B(v, \delta)}\left|v^{-1} \cdot u\right|^{-3 k} d V\left(v^{-1} \cdot u\right)\right]^{\frac{1}{k}} \\
& =L^{-1}\left\|\nabla_{b} \varphi\right\|_{4}\left[\int_{B(0, \delta)}|u|^{-3 k} d V(u)\right]^{\frac{1}{k}} \\
& =L^{-1} A_{1}^{\frac{1}{k}}\left\|\nabla_{b} \varphi\right\|_{4}\left(\int_{0}^{\delta} r^{3-3 k} d r\right)^{\frac{1}{k}} \\
& =L^{-1} A_{1}^{\frac{1}{k}} \delta^{\frac{4-3 k}{k}}\left\|\nabla_{b} \varphi\right\|_{4}\left(\frac{1}{4-3 k}\right)^{\frac{1}{k}} .
\end{aligned}
$$

Since $\frac{1}{k}=\frac{1}{p}+\frac{3}{4}$ and $p \geq 4$, we immediately get that $\left(\frac{1}{4-3 k}\right)^{\frac{1}{k}} \leq C p^{\frac{3}{4}}$ and $-3 \leq$ $\frac{4-3 k}{k} \leq 1$ for some constant $C$. Thus, by (2.4), there exists a constant $K$ such that for all $p \geq 1$,

$$
\|\varphi\|_{p} \leq K\left\|\nabla_{b} \varphi\right\|_{4} \cdot p^{\frac{3}{4}}
$$

It follows that

$$
\begin{aligned}
\int_{B} e^{\varphi} d V(u) & =\int_{B}\left(\sum_{p=0}^{\infty} \frac{\varphi^{p}}{p !}\right) d V(u) \\
& \leq \sum_{p=0}^{\infty} \frac{\|\varphi\|_{p}^{p}}{p !} \\
& \leq \sum_{p=0}^{\infty} K^{p}\left\|\nabla_{b} \varphi\right\|_{4}^{p}(p !)^{-1} p^{\frac{3 p}{4}} \\
& =\sum_{p=0}^{\infty} \frac{\left(K^{4}\right)^{\frac{p}{4}}\left(\left\|\nabla_{b} \varphi\right\|_{4}^{4}\right)^{\frac{p}{4}}}{\left(\frac{p}{4}\right) !} \frac{\left(\frac{p}{4}\right) !}{p !} p^{\frac{3 p}{4}}
\end{aligned}
$$

Here $x !=\Gamma(x+1)$ for all real number $x \geq 0$.

According to Stirling's formula, when $p \rightarrow \infty$, we can estimate 


$$
\begin{aligned}
\frac{\left(\frac{p}{4}\right) !}{p !} p^{\frac{3 p}{4}} & \approx \frac{\left(2 \pi \frac{p}{4}\right)^{\frac{1}{2}}\left(\frac{p}{4 e}\right)^{\frac{p}{4}}}{(2 \pi p)^{\frac{1}{2}}\left(\frac{p}{e}\right)^{p}} p^{\frac{3 p}{4}} \\
& \approx \frac{1}{2}\left(\frac{1}{4}\right)^{\frac{p}{4}} e^{\frac{3 p}{4}}
\end{aligned}
$$

Therefore, from (2.6), we get

$$
\begin{aligned}
\int_{B} e^{\varphi} d V(u) & \leq C \sum_{p=0}^{\infty} \frac{\left(\frac{e^{3}}{4} K^{4}\right)^{\frac{p}{4}}\left(\left\|\nabla_{b} \varphi\right\|_{4}^{4}\right)^{\frac{p}{4}}}{\left(\frac{p}{4}\right) !} \\
& \leq C \cdot \exp \left(\varkappa\left\|\nabla_{b} \varphi\right\|_{4}^{4}\right),
\end{aligned}
$$

for some constants $C$ and $\varkappa$.

Now we are ready to prove an analogue of the Moser inequality in pseudohermitian geometry. Let $C^{\infty}(M)$ denote the space of all real valued $C^{\infty}$ smooth functions on $M$.

Theorem 2.3. (Pseudohermitian Moser inequality) Let $(M, J, \theta)$ be a closed pseudohermitian 3-manifold. Then there exist constants $C, \varkappa$, and $\nu$ such that for all $\varphi \in C^{\infty}(M)$, there holds

$$
\int_{M} e^{\varphi} d \mu \leq C \exp \left(\varkappa\left\|\nabla_{b} \varphi\right\|_{4}^{4}+\nu\|\varphi\|_{4}^{4}\right)
$$

where $d \mu=\theta \wedge d \theta$ and the $L^{4}$ norm $\|\cdot\|_{4}$ respects the volume form $d \mu$.

Proof. For each point $x \in M$, there exists a neighborhood $U_{x}$ of $x$ such that $U_{x}$ is diffemorphic to a Heisenberg ball $B\left(r_{x}\right)$ centered at the origin with radius $r_{x}<1$. Choose a cut-off function $\eta_{x}$ such that $\eta_{x}(v)=1$ for $|v|<\frac{r_{x}}{2}$ and $\eta_{x}(v)=0$ for $|v| \geq r_{x}$ 
Since $M$ is closed, there exist finite balls $\left(B\left(\frac{r_{j}}{2}\right), \eta_{j}\right), j=1, \cdots m$ such that $\left\{B\left(\frac{r_{j}}{2}\right)\right\}$ is an open covering of $M$. Let $\varphi_{j}=\eta_{j} \varphi$. Making use of (2.3), we compute

$$
\begin{aligned}
\int_{M} e^{\varphi} d \mu & \leq C \cdot \sum_{j=1}^{m} \int_{B\left(\frac{r_{j}}{2}\right)} e^{\varphi} d V(u) \\
& \leq C \cdot \sum_{j=1}^{m} \int_{B} e^{\varphi_{j}} d V(u) \\
& \leq C \cdot \sum_{j=1}^{m} \exp \left(\varkappa\left\|\nabla_{b} \varphi_{j}\right\|_{4 ; B}^{4}\right) \\
& \leq C \cdot \sum_{j=1}^{m} \exp \left(\varkappa\left\|\nabla_{b} \varphi\right\|_{4}^{4}+\nu\|\varphi\|_{4}^{4}\right),
\end{aligned}
$$

for some constants $C$, $\varkappa$, and $\nu$.

\section{The Long-time Existence}

Let $T$ be the maximal time for a solution of the flow (1.6) on $M \times[0, T)$. We will derive the $S^{k, 2}$-norm estimate for $\lambda$ under the flow (1.6) for all $0 \leq t<T$. It then follows that we have the long-time existence for solutions of (1.6) on $M \times[0, \infty)$.

First we have an integral version of the Bochner formula on a pseudohermitian 3-manifold.

Lemma 3.1. Let $\left(M, J, \theta_{0}\right)$ be a closed pseudohermitian 3-manifold. Then for any $\lambda$ $\in C^{\infty}(M)$, there holds

$$
\begin{aligned}
0= & \int_{M}\left(\Delta_{0} \lambda\right)^{2} d \mu_{0}-\int_{M}\left|\nabla^{2} \lambda\right|^{2} d \mu_{0}+2 \int_{M}\left(\lambda_{0}\right)^{2} d \mu_{0} \\
& -\int_{M} \stackrel{0}{W}|\stackrel{0}{\nabla} \lambda|^{2} d \mu_{0}+2 \operatorname{Im} \int_{M} \stackrel{0}{A}_{\overline{11}} \lambda_{1} \lambda_{1} d \mu_{0}
\end{aligned}
$$

where $\stackrel{0}{\nabla}$ and $T_{0}$ denote the sub-gradient and the characteristic vector field with respect to $\left(J, \theta_{0}\right)$, respectively, and $\lambda_{0}=T_{0} \lambda$.

Proof. We first show that (see Appendix A for definitions of the notations)

$$
\begin{aligned}
& \frac{1}{2} \Delta_{0}|\stackrel{0}{\nabla} \lambda|^{2}=\left|\stackrel{0}{\nabla^{2}} \lambda\right|^{2}+<\stackrel{0}{\nabla} \lambda, \stackrel{0}{\nabla}\left(\Delta_{0} \lambda\right)>_{J, \theta_{0}}+\left.\left.\stackrel{0}{W}\right|^{\circ} \nabla\right|^{2} \\
& +\operatorname{Tor}(\stackrel{0}{\nabla} \lambda, \stackrel{0}{\nabla} \lambda)-2 i \lambda_{1} \lambda_{0 \overline{1}}+2 i \lambda_{\overline{1}} \lambda_{01} \text {, }
\end{aligned}
$$


where $\operatorname{Tor}(\stackrel{0}{\nabla} \lambda, \stackrel{0}{\nabla} \lambda) \equiv i \stackrel{0}{A_{\overline{11}}} \lambda_{1} \lambda_{1}+$ conjugate of $i \stackrel{0}{A_{\overline{11}}} \lambda_{1} \lambda_{1}$.

We compute

$$
|\stackrel{0}{\nabla} \lambda|^{2}=2\left|\lambda_{1}\right|^{2}=2 \lambda_{1} \lambda_{\overline{1}},\left.\left.\quad\right|^{0} \nabla^{2} \lambda\right|^{2}=2 \lambda_{\overline{11}} \lambda_{11}+2 \lambda_{\overline{1} 1} \lambda_{1 \overline{1}}
$$

and

$$
\begin{aligned}
\frac{1}{2} \Delta_{0}|\stackrel{0}{\nabla} \lambda|^{2} & =\left(\lambda_{1} \lambda_{\overline{1}}\right)_{1 \overline{1}}+\left(\lambda_{1} \lambda_{\overline{1}}\right)_{\overline{1} 1} \\
& =\lambda_{\overline{1} \overline{1} \overline{1}} \lambda_{1}+2 \lambda_{\overline{1} 1} \lambda_{1 \overline{1}}+2 \lambda_{\overline{11}} \lambda_{11}+\lambda_{\overline{1}} \lambda_{11 \overline{1}}+\lambda_{1} \lambda_{\overline{11} 1}+\lambda_{\overline{1}} \lambda_{1 \overline{1} 1} \\
& =\left|\nabla^{2} \lambda\right|^{2}+\lambda_{1}\left(\lambda_{\overline{11} 1}+\lambda_{\overline{1} 1 \overline{1}}\right)+\text { conjugate of } \lambda_{1}\left(\lambda_{\overline{11} 1}+\lambda_{\overline{1} 1 \overline{1}}\right) .
\end{aligned}
$$

Observe that

$$
\begin{aligned}
& <\stackrel{0}{\nabla} \lambda, \stackrel{0}{\nabla}\left(\Delta_{0} \lambda\right)>_{J, \theta_{0}}=\lambda_{1}\left(\lambda_{1 \overline{1}}+\lambda_{\overline{1} 1}\right)_{\overline{1}}+\text { conjugate of } \lambda_{1}\left(\lambda_{1 \overline{1}}+\lambda_{\overline{1} 1}\right)_{\overline{1}} \\
& =\lambda_{1}\left(\lambda_{1 \overline{11}}+\lambda_{\overline{1} 1 \overline{1}}\right)+\text { conjugate of } \lambda_{1}\left(\lambda_{1 \overline{11}}+\lambda_{\overline{1} 1 \overline{1}}\right) \text {. }
\end{aligned}
$$

Taking the covariant differentiation of

$$
\lambda_{\overline{1} 1}=\lambda_{1 \overline{1}}-i \lambda_{0}
$$

([L1] ) in the $Z_{\overline{1}}$ direction gives

$$
\lambda_{\overline{1} 1 \overline{1}}=\lambda_{1 \overline{11}}-i \lambda_{0 \overline{1}}
$$

A commutation relation ([L1] for covariant derivatives of a 1-form gives

$$
\lambda_{\overline{11} 1}=\lambda_{\overline{1} 1 \overline{1}}-i \lambda_{\overline{10}}+\stackrel{0}{W} \lambda_{\overline{1}} .
$$

Now from (3.3), (3.4), (3.6), and (3.7), we obtain

$$
\begin{aligned}
\frac{1}{2} \Delta_{0}|\stackrel{0}{\nabla} \lambda|^{2}= & \left|{ }^{2} \lambda\right|^{2}+<\stackrel{0}{\nabla} \lambda, \stackrel{0}{\nabla}\left(\Delta_{0} \lambda\right)>+\left(\stackrel{0}{W} \lambda_{1} \lambda_{\overline{1}}+\text { conjugate }\right) \\
& +\left[-i \lambda_{1}\left(\lambda_{\overline{1} 0}+\lambda_{0 \overline{1}}\right)+\text { conjugate }\right] .
\end{aligned}
$$

Then (3.2) follows from (3.8) and the following commutation relation ([L1])

$$
\lambda_{0 \overline{1}}=\lambda_{\overline{1} 0}+\stackrel{0}{A} \overline{11} \lambda_{1} .
$$

Finally integrating both sides of (3.2) and applying (3.5), we obtain

$$
\begin{aligned}
0= & \int_{M}\left(\Delta_{0} \lambda\right)^{2} d \mu_{0}-\int_{M}\left|\nabla^{2} \lambda\right|^{2} d \mu_{0}+2 \int_{M}\left(i \lambda_{1} \lambda_{0 \overline{1}}-i \lambda_{\overline{1}} \lambda_{01}\right) d \mu_{0} \\
& -\int_{M} \stackrel{0}{W}|\stackrel{0}{\nabla} \lambda|^{2} d \mu_{0}+2 \operatorname{Im} \int_{M} \stackrel{0}{A_{\overline{11}}} \lambda_{1} \lambda_{1} d \mu_{0} .
\end{aligned}
$$


By integrating by parts and (3.5), we compute

$$
\begin{aligned}
\int_{M}\left(i \lambda_{1} \lambda_{0 \overline{1}}-i \lambda_{\overline{1}} \lambda_{01}\right) d \mu_{0} & =\int_{M} i \lambda_{0}\left(-\lambda_{1 \overline{1}}+\lambda_{\overline{1} 1}\right) d \mu_{0} \\
& =\int_{M}\left(\lambda_{0}\right)^{2} d \mu_{0} .
\end{aligned}
$$

Combining the above two formulas gives (3.1).

Lemma 3.2. Let $\left(M, J, \theta_{0}\right)$ be a closed pseudohermitian 3-manifold. Then for any $\lambda$ $\in C^{\infty}(M)$, there holds

$$
\begin{aligned}
2 \int_{M} P_{0} \lambda \cdot \lambda d \mu_{0}= & 3 \int_{M}\left(\Delta_{0} \lambda\right)^{2} d \mu_{0}-\int_{M}\left|\nabla^{2} \lambda\right|^{2} d \mu_{0} \\
& -\left.\int_{M} W|\stackrel{0}{W}| \stackrel{0}{\nabla} \lambda\right|^{2} d \mu_{0}-6 \operatorname{Im} \int_{M} \stackrel{0}{A} \overline{11} \lambda_{1} \lambda_{1} d \mu_{0} .
\end{aligned}
$$

Proof. Multiplying both sides of the formula $P_{0} \lambda=\Delta_{0}^{2} \lambda+T_{0}^{2} \lambda+4 \operatorname{Im}\left(\stackrel{0}{A_{\overline{11}}} \lambda_{11}+\stackrel{0}{A_{\overline{11}, 1}} \lambda_{1}\right)$ by $\lambda$ and integrating, we compute

$$
\begin{aligned}
2 \int_{M} P_{0} \lambda \cdot \lambda d \mu_{0}= & 2 \int_{M}\left(\Delta_{0} \lambda\right)^{2} d \mu_{0}-2 \int_{M}\left(\lambda_{0}\right)^{2} d \mu_{0}-8 \operatorname{Im} \int_{M} \stackrel{0}{A} \overline{11} \lambda_{1} \lambda_{1} d \mu_{0} \\
= & 3 \int_{M}\left(\Delta_{0} \lambda\right)^{2} d \mu_{0}-\int_{M}\left|\stackrel{0}{\nabla}^{2} \lambda\right|^{2} d \mu_{0}-\int_{M} \stackrel{0}{W}|\stackrel{0}{\nabla} \lambda|^{2} d \mu_{0} \\
& -6 \operatorname{Im} \int_{M} \stackrel{0}{A}_{\overline{11}} \lambda_{1} \lambda_{1} d \mu_{0}
\end{aligned}
$$

by integrating by parts and Lemma 3.1 .

As a consequence of Lemma 3.2 and (1.11), we have

Corollary 3.3. Let $\left(\mathbf{S}^{3}, J, \theta_{0}\right)$ be the standard pseudohermitian 3-sphere. Then

$$
\int_{\mathbf{S}^{3}}\left|\nabla^{2} \lambda\right|^{2} d \mu_{0} \leq 2 \int_{\mathbf{S}^{3}}\left(\Delta_{0} \lambda\right)^{2} d \mu_{0}
$$

for $\lambda \perp \operatorname{ker}\left(P_{0}\right)$.

It follows from (1.7) that

Lemma 3.4. Let $\left(M, J,\left[\theta_{0}\right]\right)$ be a closed $C R$ 3-manifold. Let $\lambda$ be a solution of the flow (1.6) on $M \times[0, T)$. Then there exists a positive constant $\beta=\beta\left(Q_{0}, \theta_{0}\right)$ such 
that

$$
\mathcal{E}(\theta)=\int_{M} P_{0} \lambda \cdot \lambda d \mu_{0}+\int_{M} Q_{0} \lambda d \mu_{0} \leq \beta^{2}
$$

for all $t \in[0, T)$.

Lemma 3.5. Let $f:[0, T) \rightarrow R$ be a $C^{1}$ smooth function satisfying $f^{\prime} \leq-C_{1} f+C_{2}$ for some positive constants $C_{1}, C_{2}>0$. Then $f(t) \leq f(0) e^{-C_{1} t}+\frac{C_{2}}{C_{1}}$ for $t \in[0, T)$.

We will often use the above lemma (whose proof is left to the reader) to obtain the higher order estimates. Now we write $\lambda=\lambda_{\text {ker }}+\lambda^{\perp} . Q_{0}=\left(Q_{0}\right)_{\text {ker }}+Q_{0}^{\perp}$ with respect to $P_{0}$. Comparing both sides of the following formula:

$$
\frac{\partial \lambda_{\mathrm{ker}}}{\partial t}+\frac{\partial \lambda^{\perp}}{\partial t}=\frac{\partial \lambda}{\partial t}=-\left(Q_{0}+2 P_{0} \lambda\right)+r(t)
$$

we obtain

$$
\frac{\partial \lambda^{\perp}}{\partial t}=-\left(Q_{0}^{\perp}+2 P_{0} \lambda^{\perp}\right)
$$

and

$$
\frac{\partial \lambda_{\mathrm{ker}}}{\partial t}=-\left(Q_{0}\right)_{\mathrm{ker}}+r(t)
$$

From now on, $C$ or $C_{j}(C(k, T), C(T)$, etc., respectively) denotes a generic constant (with emphasis on depending on $(k, T), T$, etc., respectively) which may vary from line to line.

Proposition 3.6. Let $\left(M, J,\left[\theta_{0}\right]\right)$ be a closed $C R$ 3-manifold with $\stackrel{0}{A} A_{11}=0$. Then under the flow (1.6) (or equivalently (3.10) and (3.11)), there exists a positive constant $C\left(k, \Upsilon, \theta_{0}, \beta, T\right)>0$ such that

$$
\|\lambda\|_{S^{2 k, 2}} \leq C\left(k, \Upsilon, \theta_{0}, \beta, T\right)
$$

for all $0 \leq t<T$.

Proof. From (3.11), we have

$$
\lambda_{\text {ker }}(t, p)-\lambda_{\text {ker }}(0, p)=-\left(Q_{0}\right)_{\text {ker }}(p) t+\int_{0}^{t} r d t .
$$


Since $\left(P_{0}\right.$ being self-adjoint)

$$
\int_{M} P_{0} \lambda^{\perp} \cdot \lambda_{\mathrm{ker}} d \mu_{0}=0
$$

and

$$
\begin{aligned}
& \int_{M} Q_{0} \lambda_{\mathrm{ker}} d \mu_{0} \\
& =\int_{M} Q_{0}\left[\lambda_{\mathrm{ker}}(0, p)-\left(Q_{0}\right)_{\mathrm{ker}}(p) t+\int_{0}^{t} r d t\right] d \mu_{0} \\
& =\int_{M} Q_{0} \lambda_{\mathrm{ker}}(0, p) d \mu_{0}-t \int_{M}\left[\left(Q_{0}\right)_{\mathrm{ker}}(p)\right]^{2} d \mu_{0} \\
& \geq-C-C t
\end{aligned}
$$

we compute

$$
\begin{aligned}
& \int_{M} P_{0} \lambda \cdot \lambda d \mu_{0}+\int_{M} Q_{0} \lambda d \mu_{0} \\
& =\int_{M} P_{0} \lambda^{\perp} \cdot\left(\lambda_{\mathrm{ker}}+\lambda^{\perp}\right) d \mu_{0}+\int_{M} Q_{0}\left(\lambda_{\mathrm{ker}}+\lambda^{\perp}\right) d \mu_{0} \\
& \geq \int_{M} P_{0} \lambda^{\perp} \cdot \lambda^{\perp} d \mu_{0}+\int_{M} Q_{0} \lambda^{\perp} d \mu_{0}-C-C t .
\end{aligned}
$$

It then follows from Lemma 3.4 that

$$
\int_{M} P_{0} \lambda^{\perp} \cdot \lambda^{\perp} d \mu_{0}+\int_{M} Q_{0} \lambda^{\perp} d \mu_{0} \leq\left(\beta^{2}+C\right)+C t
$$

for all $t \in[0, T)$. Since the torsion of $\left(M, J, \theta_{0}\right)$ vanishes, the CR Paneitz operator $P_{0}$ is essentially positive in the sense that

$$
\int_{M} P_{0} \lambda^{\perp} \cdot \lambda^{\perp} d \mu_{0} \geq \Upsilon \int_{M}\left(\lambda^{\perp}\right)^{2} d \mu_{0}
$$

by Theorem 5.3. All these imply that there exists a positive constant $C\left(\Upsilon, Q_{0}, \theta_{0}\right)>0$ such that

$$
\begin{aligned}
\left(\beta^{2}+C\right)+C t & \geq \int_{M} P_{0} \lambda^{\perp} \cdot \lambda^{\perp} d \mu_{0}+\int_{M} Q_{0} \lambda^{\perp} d \mu_{0} \\
& \geq \frac{\Upsilon}{2} \int_{M}\left(\lambda^{\perp}\right)^{2} d \mu_{0}-C\left(\Upsilon, Q_{0}, \theta_{0}\right)
\end{aligned}
$$

for all $t \in[0, T)$. So there exists a positive constant $C\left(\Upsilon, Q_{0}, \theta_{0}, \beta, T\right)$ such that

$$
\int_{M}\left(\lambda^{\perp}\right)^{2} d \mu_{0} \leq C\left(\Upsilon, Q_{0}, \theta_{0}, \beta, T\right)
$$

for all $t \in[0, T)$.

On the other hand, we observe from the condition $\stackrel{0}{A_{11}}=0$ that

$$
\Delta_{0}^{k} T_{0}^{2}=T_{0}^{2} \Delta_{0}^{k}
$$


and hence

$$
\Delta_{0}^{k} P_{0}=P_{0} \Delta_{0}^{k}
$$

It follows that

$$
\Delta_{0}^{k} \lambda^{\perp} \perp \operatorname{ker} P_{0}, \Delta_{0}^{k} \lambda_{\text {ker }} \in \operatorname{ker} P_{0}
$$

Next we compute, for all positive integers $k$,

$$
\begin{aligned}
& \frac{d}{d t} \int_{M}\left(\Delta_{0}^{k} \lambda^{\perp}\right)^{2} d \mu_{0} \\
& =2 \int_{M}\left(\Delta_{0}^{k} \lambda^{\perp}\right)\left(\Delta_{0}^{k} \frac{\partial \lambda^{\perp}}{\partial t}\right) d \mu_{0} \\
& =-2 \int_{M}\left(\Delta_{0}^{k} \lambda^{\perp}\right)\left[\Delta_{0}^{k}\left(Q_{0}^{\perp}+2 P_{0} \lambda^{\perp}\right)\right] d \mu_{0} \\
& =-2 \int_{M} \lambda^{\perp}\left[\Delta_{0}^{2 k}\left(Q_{0}^{\perp}\right)\right] d \mu_{0}-4 \int_{M}\left(\Delta_{0}^{k} \lambda^{\perp}\right)\left(P_{0} \Delta_{0}^{k} \lambda^{\perp}\right) d \mu_{0} .
\end{aligned}
$$

Here we have used (3.15). By (3.16) and essential positivity of $P_{0}$, we obtain

$$
\int_{M}\left(\Delta_{0}^{k} \lambda^{\perp}\right)\left(P_{0} \Delta_{0}^{k} \lambda^{\perp}\right) d \mu_{0} \geq \Upsilon \int_{M}\left(\Delta_{0}^{k} \lambda^{\perp}\right)^{2} d \mu_{0}
$$

Therefore from (3.17), (3.18), and (3.13), we conclude that

$$
\frac{d}{d t} \int_{M}\left(\Delta_{0}^{k} \lambda^{\perp}\right)^{2} d \mu_{0} \leq-4 \Upsilon \int_{M}\left(\Delta_{0}^{k} \lambda^{\perp}\right)^{2} d \mu_{0}+C(k, T) .
$$

By applying Lemma 3.5 to the O.D.E. $f^{\prime}(t) \leq-4 \Upsilon f(t)+C(k, T)$ from (3.19), we obtain

$$
\int_{M}\left(\Delta_{0}^{k} \lambda^{\perp}\right)^{2} d \mu_{0} \leq C(k, T)
$$

and hence

$$
\left\|\lambda^{\perp}-\overline{\lambda^{\perp}}\right\|_{S^{2 k, 2}} \leq C(k, T)
$$

for all $0 \leq t<T$.

From the definition of the $Q$-curvature, ${ }^{A} A_{11}=0$, and (3.16), we compute

$$
\int_{M}\left(Q_{0}\right)_{\operatorname{ker}} d \mu_{0}=-\frac{2}{3} \int_{M} \Delta_{0}\left(R_{0}\right)_{\mathrm{ker}} d \mu_{0}=0 .
$$

This and (3.12) imply

$$
\lambda-\bar{\lambda}=\left(\lambda^{\perp}-\overline{\lambda^{\perp}}\right)+\left[\lambda_{\mathrm{ker}}(0, p)-\left(Q_{0}\right)_{\mathrm{ker}}(p) t\right]-\overline{\lambda_{\mathrm{ker}}(0, p)},
$$


and hence

$$
\|\lambda-\bar{\lambda}\|_{S^{2 k, 2}} \leq C\left(\left(Q_{0}\right)_{\mathrm{ker}}, k, T\right)+\left\|\lambda^{\perp}-\overline{\lambda^{\perp}}\right\|_{S^{2 k, 2}} \leq C(k, T)
$$

for all $0 \leq t<T$. Recall that the average $\bar{f}$ of a function $f$ is defined by $\bar{f}=\frac{\int_{M} f d \mu_{0}}{\int_{M} d \mu_{0}}$. In particular, there holds

$$
\|\lambda-\bar{\lambda}\|_{S^{2,2}} \leq C(T)
$$

Therefore by the Sobolev embedding theorem, we have $S^{2,2} \subset S^{1,4}$ and

$$
\|\lambda-\bar{\lambda}\|_{S^{1,4}} \leq C(T)
$$

Now using Theorem 2.3 (pseudohermitian Moser inequality), we get

$$
\int_{M} e^{4(\lambda-\bar{\lambda})} d \mu_{0} \leq C \exp \left(C\|\lambda-\bar{\lambda}\|_{S^{1,4}}\right) \leq C(T)
$$

Together with $\int_{M} e^{4 \lambda} d \mu_{0}$ being invariant under the flow, we conclude that

$$
C \geq \bar{\lambda} \geq-C(T)
$$

(the upper bound is obtained by observing that $\int \lambda d \mu_{0} \leq \int e^{4 \lambda} d \mu_{0}$ ). By (3.20) and (3.22), we finally obtain

$$
\|\lambda\|_{S^{2 k, 2}} \leq C(k, T)
$$

for all $0 \leq t<T$.

\section{Proof of Theorem 1.1;}

When the torsion is zero, we can write $P_{0}=\square_{0} \overline{\square_{0}}=\overline{\square_{0}} \square_{0}$ ( $\square_{0}$ and $\overline{\square_{0}}$ commute) where $\square_{0} \equiv \Delta_{0}+i T_{0}$ is the Kohn Laplacian (acting on functions) with respect to $\left(J, \theta_{0}\right)$, and $T_{0}$ is an infinitesimal $C R$ diffeomorphism. This implies that $(M, J)$ is embeddable (see Theorem 2.1 in $\left[\mathrm{Le}\right.$ ). Therefore $\square_{0}$ and $\square_{0}$ have subelliptic estimates on the orthogonal complements of $\operatorname{Ker}\left(\square_{0}\right)$ and $\operatorname{Ker}\left(\overline{\square_{0}}\right)$ in $L^{2}$, denoted as $\left(\operatorname{Ker}\left(\square_{0}\right)\right)^{\perp}$ and $\left(\operatorname{Ker}\left(\overline{\square_{0}}\right)\right)^{\perp}$, respectively $([\underline{K} 1]$, [K2 $)$. Since $P_{0}$ commutes with $\square_{0}$ and $\operatorname{Ker}\left(\overline{\square_{0}}\right) \subset \operatorname{Ker} P_{0}$, we have

$$
\square_{0} \varphi \in\left(\operatorname{Ker} P_{0}\right)^{\perp} \subset\left(\operatorname{Ker}\left(\overline{\square_{0}}\right)\right)^{\perp}
$$

for $\varphi \in\left(\operatorname{Ker} P_{0}\right)^{\perp}$. We can then estimate 


$$
\begin{aligned}
\|\varphi\|_{S^{k+4,2}} & \leq C\left\|\square_{0} \varphi\right\|_{S^{k+2,2}} \\
& \leq C\left\|\square_{0} \square_{0} \varphi\right\|_{S^{k, 2}}=C\left\|P_{0} \varphi\right\|_{S^{k, 2}}
\end{aligned}
$$

for $\varphi \in\left(\operatorname{Ker} P_{0}\right)^{\perp}\left(\subset\left(\operatorname{Ker}\left(\square_{0}\right)\right)^{\perp}\right)$ by (3.23). By (B.24) , we mean that $P_{0}$ is subelliptic on $\left(\operatorname{Ker} P_{0}\right)^{\perp}$. It follows that there exists a unique $C^{\infty}$ smooth solution $\lambda^{\perp}$ of (3.10) for a short time (noting that $P_{0}$ is essentially positive by Theorem [5.3, which explains the negative sign in (3.10) ). On the other hand, we can apply the contraction mapping

principle to show the short time existence of a unique $C^{\infty}$ smooth solution $\lambda_{\mathrm{ker}}$ to (3.11) (observing that $\lambda_{\mathrm{ker}}$ satisfies (3.12)). The long time solution then follows from Proposition 3.6, the Sobolev embedding theorem for $S^{k, 2}$, and the standard argument for extending the solution at the maximal time $T$.

\section{A Priori Estimates and Asymptotic Convergence}

We can obtain an a priori $S^{2,2}$ estimate under some analytic assumption replacing the torsion free condition. Also a condition on the background $Q$-curvature will assure that the bound is uniform, i.e., independent of the time. Under the same condition together with the vanishing torsion, we will then have the higher-order estimates with unform bounds. Therefore we are able to prove the asymptotic convergence of solutions of (1.6).

Lemma 4.1. Suppose $\left(Q_{0}\right)_{\mathrm{ker}}=0$. Then the solution $\lambda^{\perp}$ of (3.10) satisfies the following a priori estimate

$$
\mathcal{E}\left(\lambda^{\perp}\right) \equiv \int_{M} P_{0} \lambda^{\perp} \cdot \lambda^{\perp} d \mu_{0}+\int_{M} Q_{0} \lambda^{\perp} d \mu_{0} \leq \beta^{2}
$$

for a constant $\beta$ independent of the time.

Proof. The condition $\left(Q_{0}\right)_{\mathrm{ker}}=0$ implies that

$$
\int_{M} Q_{0} \lambda_{\mathrm{ker}} d \mu_{0}=0
$$


It follows that

$$
\begin{aligned}
& \int_{M} P_{0} \lambda \cdot \lambda d \mu_{0}+\int_{M} Q_{0} \lambda d \mu_{0} \\
& =\int_{M} P_{0} \lambda^{\perp} \cdot\left(\lambda_{\mathrm{ker}}+\lambda^{\perp}\right) d \mu_{0}+\int_{M} Q_{0}\left(\lambda_{\mathrm{ker}}+\lambda^{\perp}\right) d \mu_{0} \\
& =\int_{M} P_{0} \lambda^{\perp} \cdot \lambda^{\perp} d \mu_{0}+\int_{M} Q_{0} \lambda^{\perp} d \mu_{0} .
\end{aligned}
$$

Hence from Lemma 3.4, we obtain (4.1).

\section{Proof of Theorem 1.2:}

The essential positivity of $P_{0}$ assures that for some constant $\Upsilon$, there holds

$$
\int_{M} P_{0} \lambda^{\perp} \cdot \lambda^{\perp} d \mu_{0} \geq \Upsilon \int_{M}\left(\lambda^{\perp}\right)^{2} d \mu_{0}
$$

From the proof of Proposition [3.6, we have (3.13), i.e., a bound for the $L^{2}$ norm of $\lambda^{\perp}$, depending on the maximal time $T$. If in addition we assume $\left(Q_{0}\right)_{\mathrm{ker}}=0$, then by (4.1), (4.2), and the Young inequality, we obtain

$$
\int_{M}\left(\lambda^{\perp}\right)^{2} d \mu_{0} \leq C\left(\Upsilon, Q_{0}, \theta_{0}, \beta\right)
$$

and

$$
\int_{M} P_{0} \lambda \cdot \lambda d \mu_{0}=\int_{M} P_{0} \lambda^{\perp} \cdot \lambda^{\perp} d \mu_{0} \leq C\left(\Upsilon, Q_{0}, \theta_{0}, \beta\right)
$$

for all $t \geq 0$.

Now if the condition $(*)$ holds on $\left(M, J,\left[\theta_{0}\right]\right)$, that is,

$$
\int_{M}\left|\nabla^{2} \lambda^{\perp}\right|^{2} d \mu_{0} \leq\left(2+\varepsilon_{0}\right) \int_{M}\left(\Delta_{0} \lambda^{\perp}\right)^{2} d \mu_{0}+C\left(\varepsilon_{0}\right) \int_{M}\left|\stackrel{0}{\nabla} \lambda^{\perp}\right|^{2} d \mu_{0} .
$$

¿From this and Lemma 3.2, we have

$$
2 \int_{M} P_{0} \lambda^{\perp} \cdot \lambda^{\perp} d \mu_{0} \geq\left(1-\varepsilon_{0}\right) \int_{M}\left(\Delta_{0} \lambda^{\perp}\right)^{2} d \mu_{0}-C \int_{M}\left|\nabla^{0} \lambda^{\perp}\right|^{2} d \mu_{0} .
$$

By applying (4.3), integrating by parts and the Young inequality, we compute

$$
\begin{aligned}
2 \int_{M} P_{0} \lambda^{\perp} \cdot \lambda^{\perp} d \mu_{0} & =\left(1-\varepsilon_{0}\right) \int_{M}\left(\Delta_{0} \lambda^{\perp}\right)^{2} d \mu_{0}+C \int_{M} \lambda^{\perp} \Delta_{0} \lambda^{\perp} d \mu_{0} \\
& \geq\left(1-\varepsilon_{0}-\varepsilon\right) \int_{M}\left(\Delta_{0} \lambda^{\perp}\right)^{2} d \mu_{0}-C\left(\varepsilon_{0}, \frac{1}{\varepsilon}\right)
\end{aligned}
$$


for small $\varepsilon>0$. Now if we choose $\varepsilon$ small enough such that $\left(1-\varepsilon_{0}-\varepsilon\right)=\delta>0$, then

$$
2 \int_{M} P_{0} \lambda^{\perp} \cdot \lambda^{\perp} d \mu_{0} \geq \delta \int_{M}\left(\Delta_{0} \lambda^{\perp}\right)^{2} d \mu_{0}-C\left(\varepsilon_{0}, \frac{1}{\varepsilon}\right) .
$$

Finally from (4.4) and (4.6), there exists a positive constant $C=C\left(\delta, \Upsilon, Q_{0}, \theta_{0}, \beta\right)$ such that

$$
\int_{M}\left(\Delta_{0} \lambda^{\perp}\right)^{2} d \mu_{0} \leq C
$$

and hence

$$
\left\|\lambda^{\perp}\right\|_{S^{2,2}} \leq C
$$

for all $t \geq 0$ by (4.3) (the constant $C$ depends on the maximal time $T$ by (3.13) if we don't assume $\left.\left(Q_{0}\right)_{\mathrm{ker}}=0\right)$.

To deal with $\lambda_{\mathrm{ker}}$, we consider $\lambda-\bar{\lambda}$. Without the condition $\left(Q_{0}\right)_{\mathrm{ker}}=0$, we have obtained the bound for $\|\lambda\|_{S^{2,2}}$, depending on $T$, from the later part of the proof of Proposition 3.6. Let us assume $\left(Q_{0}\right)_{\text {ker }}=0$ below and see how to get an uniform bound.

Under the condition $\left(Q_{0}\right)_{\text {ker }}=0$, the equation (3.11) reads

$$
\frac{\partial \lambda_{\text {ker }}}{\partial t}=r
$$

and hence we have

$$
\lambda_{\mathrm{ker}}(t, p)=\lambda_{\mathrm{ker}}(0, p)+\int_{0}^{t} r d t .
$$

It follows that

$$
\lambda-\bar{\lambda}=\left(\lambda^{\perp}-\overline{\lambda^{\perp}}\right)+\left[\lambda_{\text {ker }}(0, p)\right]
$$

and from (4.7) and (4.3) that

$$
\|\lambda-\bar{\lambda}\|_{S^{2,2}} \leq C_{1}+\left\|\lambda^{\perp}-\overline{\lambda^{\perp}}\right\|_{S^{2,2}} \leq C_{2}
$$

for all $t \geq 0$. Therefore by the Sobolev embedding theorem for $S^{k, p}$, we have

$$
\|\lambda-\bar{\lambda}\|_{S^{1,4}} \leq C_{3}
$$

for all $t \geq 0$. 
Now again by using Theorem 2.3, we have

$$
\int_{M} e^{4(\lambda-\bar{\lambda})} d \mu_{0} \leq C \exp \left(C\|\lambda-\bar{\lambda}\|_{S^{1,4}}\right) \leq C_{4}
$$

for all $t \geq 0$. Together with $\int_{M} e^{4 \lambda} d \mu_{0}$ being invariant under the flow, we conclude that

$$
C_{5} \geq \bar{\lambda} \geq-C_{5}
$$

Thus

$$
\|\lambda\|_{S^{2,2}} \leq C
$$

(and also (from Theorem 2.3)

$$
\int_{M} e^{\alpha \lambda} d \mu_{0} \leq C
$$

for all real number $\alpha$ and all $t \geq 0$ ).

Since $\Delta_{0}$ is self adjoint, we can deduce the following result by the Young inequality.

Lemma 4.2. (interpolation inequality for $\Delta_{0}$ ) On a closed pseudohermitian manifold $\left(M, J, \theta_{0}\right)$, given $\varepsilon>0$, there exists a constant $C(\varepsilon)$ such that

$$
\int_{M}\left(\Delta_{0} \varphi\right)^{2} d \mu_{0} \leq \varepsilon \int_{M}\left(\Delta_{0}^{2} \varphi\right)^{2} d \mu_{0}+C(\varepsilon) \int_{M} \varphi^{2} d \mu_{0}
$$

for all real valued functions $\varphi \in C^{\infty}(M)$.

\section{Proof of Theorem 1.3:}

We compute

$$
\begin{aligned}
& \frac{d}{d t} \int_{M}\left(\Delta_{0}^{k} \lambda^{\perp}\right)^{2} d \mu_{0} \\
& =2 \int_{M}\left(\Delta_{0}^{k} \lambda^{\perp}\right)\left(\Delta_{0}^{k} \frac{\partial \lambda^{\perp}}{\partial t}\right) d \mu_{0} \\
& =-2 \int_{M}\left(\Delta_{0}^{k} \lambda^{\perp}\right)\left[\Delta_{0}^{k}\left(Q_{0}+2 P_{0} \lambda^{\perp}\right)\right] d \mu_{0} \quad(\text { by (3.10) }) \\
& =-2 \int_{M}\left(\Delta_{0}^{k} \lambda^{\perp}\right)\left[\Delta_{0}^{k}\left(Q_{0}\right)\right] d \mu_{0}-4 \int_{M}\left(\Delta_{0}^{k} \lambda^{\perp}\right)\left[\Delta_{0}^{k}\left(P_{0} \lambda^{\perp}\right)\right] d \mu_{0} .
\end{aligned}
$$


Next we will estimate the second term in the last line of (4.13). From the Bochner formula (3.1), (3.16), and (3.14), we compute

$$
\begin{aligned}
& -4 \int_{M}\left(\Delta_{0}^{k} \lambda^{\perp}\right)\left[\Delta_{0}^{k}\left(P_{0} \lambda^{\perp}\right)\right] d \mu_{0} \\
& =-4 \int_{M}\left(\Delta_{0}^{k} \lambda^{\perp}\right)\left[\Delta_{0}^{k}\left(\Delta_{0}^{2} \lambda^{\perp}+T_{0}^{2} \lambda^{\perp}\right)\right] d \mu_{0}+E_{1} \\
& =-4 \int_{M}\left(\Delta_{0}^{k+1} \lambda^{\perp}\right)^{2} d \mu_{0}-4 \int_{M}\left(\Delta_{0}^{k} \lambda^{\perp}\right)\left[\Delta_{0}^{k} T_{0}^{2} \lambda^{\perp}\right] d \mu_{0}+E_{1} \\
& =-4 \int_{M}\left(\Delta_{0}^{k+1} \lambda^{\perp}\right)^{2} d \mu_{0}-4 \int_{M}\left(\Delta_{0}^{k} \lambda^{\perp}\right)\left[T_{0}^{2} \Delta_{0}^{k} \lambda^{\perp}\right] d \mu_{0}+E_{1}+E_{2} \\
& =-4 \int_{M}\left(\Delta_{0}^{k+1} \lambda^{\perp}\right)^{2} d \mu_{0}+4 \int_{M}\left(T_{0} \Delta_{0}^{k} \lambda^{\perp}\right)^{2} d \mu_{0}+E_{1}+E_{2} \\
& =2\left[\int_{M}\left|\nabla^{2}\left(\Delta_{0}^{k} \lambda^{\perp}\right)\right|^{2} d \mu_{0}-3 \int_{M}\left(\Delta_{0}^{k+1} \lambda^{\perp}\right)^{2} d \mu_{0}\right]+E_{1}+E_{2}+E_{3}
\end{aligned}
$$

where

$$
\begin{aligned}
& E_{1}=\int_{M}\left(\Delta_{0}^{k} \lambda^{\perp}\right) \Delta_{0}^{k}\left(O_{2}\left(\lambda^{\perp}\right)\right) d \mu_{0}, \\
& \left.E_{2}=\int_{M}\left(\Delta_{0}^{k} \lambda^{\perp}\right) O_{2 k}\left(\lambda^{\perp}\right)\right) d \mu_{0}, \\
& E_{3}=\int_{M}\left(\Delta_{0}^{k} \lambda^{\perp}\right) O_{2 k+2}\left(\lambda^{\perp}\right) d \mu_{0},
\end{aligned}
$$

with $O_{j}$ being some differential operator of weight $j$ (see Appendix $A$ for the definition). Using the subelliptic estimate and the Young inequality, we can estimate the "error terms" $E_{j}^{\prime} s$ by

$$
\begin{aligned}
& |E| \\
& =\left|E_{1}+E_{2}+E_{3}\right| \\
& \leq C\left(k, \varepsilon_{1}\right) \int_{M}\left(\Delta_{0}^{k} \lambda^{\perp}\right)^{2} d \mu_{0}+\varepsilon_{1}\left(\int_{M}\left(\Delta_{0}^{k+1} \lambda^{\perp}\right)^{2} d \mu_{0}+\int_{M}\left(\lambda^{\perp}\right)^{2} d \mu_{0}\right) .
\end{aligned}
$$

Note that $\Delta_{0}$ (and hence $\Delta_{0}^{k}$ ) maps $\operatorname{Ker}\left(P_{0}\right)$, and hence $\operatorname{Ker}\left(P_{0}\right)^{\perp}$, into itself by assumption. So we can apply the condition $(*)$ to the first term of the last line in (4.14). Together with substituting (4.15) into (4.14), we obtain

$$
\begin{aligned}
& -4 \int_{M}\left(\Delta_{0}^{k} \lambda^{\perp}\right)\left[\Delta_{0}^{k}\left(P_{0} \lambda^{\perp}\right)\right] d \mu_{0} \\
& \leq-2\left(1-\varepsilon_{0}-\frac{\varepsilon_{1}}{2}\right) \int_{M}\left(\Delta_{0}^{k+1} \lambda^{\perp}\right)^{2} d \mu_{0}+C\left(k, \varepsilon_{1}\right) \int_{M}\left(\Delta_{0}^{k} \lambda^{\perp}\right)^{2} d \mu_{0}+\varepsilon_{1} \int_{M}\left(\lambda^{\perp}\right)^{2} d \mu_{0} .
\end{aligned}
$$

Taking $\varepsilon_{1}$ small so that $1-\varepsilon_{0}-\frac{\varepsilon_{1}}{2}>0$. From the interpolation inequality (4.12) with $\varphi=\Delta_{0}^{k-1} \lambda^{\perp}$, we get

$$
\int_{M}\left(\Delta_{0}^{k} \lambda^{\perp}\right)^{2} d \mu_{0} \leq \varepsilon \int_{M}\left(\Delta_{0}^{k+1} \lambda^{\perp}\right)^{2} d \mu_{0}+C(\varepsilon) \int_{M}\left(\Delta_{0}^{k-1} \lambda^{\perp}\right)^{2} d \mu_{0}
$$


By choosing $\varepsilon$ (depending on $k, \varepsilon_{1}$ ) small enough, we can absorb the middle term of the second line into the first term of the second line in (4.16). Substituting (4.17) into (4.16) and then substituting the result into (4.13), we obtain

$$
\begin{aligned}
& \frac{d}{d t} \int_{M}\left(\Delta_{0}^{k} \lambda^{\perp}\right)^{2} d \mu_{0} \\
& \leq-C \int_{M}\left(\Delta_{0}^{k+1} \lambda^{\perp}\right)^{2} d \mu_{0}+C(k) \int_{M}\left(\Delta_{0}^{k-1} \lambda^{\perp}\right)^{2} d \mu_{0}+C \int_{M}\left(\lambda^{\perp}\right)^{2} d \mu_{0} .
\end{aligned}
$$

In view of the subellipticity of $\Delta_{0}^{k}$, we have

$$
\int_{M}\left(\Delta_{0}^{k} \lambda^{\perp}\right)^{2} d \mu_{0} \leq C(k)\left(\int_{M}\left(\Delta_{0}^{k+1} \lambda^{\perp}\right)^{2} d \mu_{0}+\int_{M}\left(\lambda^{\perp}\right)^{2} d \mu_{0}\right) .
$$

Therefore we can reduce (4.18) to

$$
\begin{aligned}
& \frac{d}{d t} \int_{M}\left(\Delta_{0}^{k} \lambda^{\perp}\right)^{2} d \mu_{0} \\
\leq & -C(k) \int_{M}\left(\Delta_{0}^{k} \lambda^{\perp}\right)^{2} d \mu_{0}+C(k) \int_{M}\left(\Delta_{0}^{k-1} \lambda^{\perp}\right)^{2} d \mu_{0}+C(k) \int_{M}\left(\lambda^{\perp}\right)^{2} d \mu_{0} .
\end{aligned}
$$

Now we apply induction on $k \geq 2$ to (4.18). When $k=2$, the last two terms of (4.19) are bounded due to Theorem 1.2. So by Lemma 3.5, we get

$$
\int_{M}\left(\Delta_{0}^{2} \lambda^{\perp}\right)^{2} d \mu_{0} \leq C(T)
$$

and $C(T)$ is independent of $T$ if the condition $\left(Q_{0}\right)_{\mathrm{ker}}=0$ is imposed. By induction hypothesis, $\int_{M}\left(\Delta_{0}^{k-1} \lambda^{\perp}\right)^{2} d \mu_{0}$ is bounded. Also recall in the proof of Proposition 3.6 that the essential positivity of $P_{0}$ and the energy estimate (3.9) imply the $L^{2}$ bound of $\lambda^{\perp}$ (see (3.13) and (4.3) if, in addition, $\left(Q_{0}\right)_{\text {ker }}=0$ ). So the last two terms of (4.19) are bounded. Applying Lemma 3.5 again, we conclude that

$$
\int_{M}\left(\Delta_{0}^{k} \lambda^{\perp}\right)^{2} d \mu_{0} \leq C(k, T)
$$

where $C(k, T)$ is independent of $T$ if the condition $\left(Q_{0}\right)_{\mathrm{ker}}=0$ is imposed. From (3.13) (4.3) if, in addition, $\left.\left(Q_{0}\right)_{\mathrm{ker}}=0\right)$, (4.20), and the subelliptic estimate, we can then have

$$
\left\|\lambda^{\perp}\right\|_{S^{2 k, 2}} \leq C(k, T)
$$


$\left(C(k, T)\right.$ is independent of $T$ if the condition $\left(Q_{0}\right)_{\mathrm{ker}}=0$ is imposed). Observe that the argument from (3.20) to (3.22) in the proof of Proposition 3.6 still works without torsion free condition. We therefore have the following estimate

$$
\|\lambda\|_{S^{2 k, 2}} \leq C(k, T)
$$

with $C(k, T)$ being independent of $T$ if the condition $\left(Q_{0}\right)_{\text {ker }}=0$ is imposed.

Proposition 4.3. Let $\left(M, J,\left[\theta_{0}\right]\right)$ be a CR 3-manifold with $\stackrel{0}{A_{11}}=0$ and $\left(Q_{0}\right)_{\mathrm{ker}}=0$. There exists a constant $C=C\left(\Upsilon, Q_{0}, \theta_{0}, \beta\right)$ such that

$$
\|\lambda\|_{S^{4,2}} \leq C
$$

for all $t \geq 0$. In particular, there holds

$$
|\lambda| \leq C
$$

for all $t \geq 0$. Moreover, we have

$$
\|\lambda\|_{S^{2 k, 2}} \leq C(k)
$$

for all $t \geq 0$.

Proof. Observe that $\lambda$ has an uniform $L^{2}$ bound if $\left(Q_{0}\right)_{\mathrm{ker}}=0$ in the proof of Proposition (3.6), and hence the constants $C(\ldots, T), C(T)$, and $C(k, T)$ can be replaced by $C$ or $C(k)$ (independent of $T$ ) from (3.13) to the end of the proof.

\section{Proof of Theorem 1.4:}

By Theorem 1.1, we have a long time solution. Starting from (1.7), we compute

$$
\begin{aligned}
\frac{d^{2}}{d t^{2}} \mathcal{E}(\theta) & =-4 \int\left(Q_{0}+2 P_{0} \lambda\right) P_{0} \frac{\partial \lambda}{\partial t} d \mu_{0} \\
& \left.=4 \int\left(Q_{0}^{\perp}+2 P_{0} \lambda\right) P_{0}\left(Q_{0}^{\perp}+2 P_{0} \lambda\right) d \mu_{0} \text { by (1.6) }\right) \\
& \geq 4 \Upsilon \int\left(Q_{0}^{\perp}+2 P_{0} \lambda\right)^{2}
\end{aligned}
$$

by essential positivity of $P_{0}$ (which is implied by the torsion free condition; see Theorem 5.3). Therefore $\frac{d}{d t} \mathcal{E}(\theta)$ is nondecreasing, and hence

$$
\int_{M} e^{4 \lambda} Q^{2} d \mu(\geq 0) \text { is nonincreasing. }
$$


By (4.21), we can find a sequence of times $t_{j}$ such that $\lambda_{j} \equiv \lambda\left(\cdot, t_{j}\right)$ converges to $\lambda_{\infty}$ in $C^{\infty}$ topology as $t_{j} \rightarrow \infty$. On the other hand, integrating (1.7) gives

$$
\mathcal{E}\left(\lambda_{\infty}\right)-\mathcal{E}\left(\lambda_{0}\right)=-\int_{0}^{\infty} \int_{M} e^{4 \lambda} Q^{2} d \mu d t
$$

In view of (4.23), (4.24), we obtain

$$
0=\lim _{t \rightarrow \infty} \int_{M} e^{4 \lambda} Q^{2} d \mu=\int_{M} e^{4 \lambda_{\infty}} Q_{\infty}^{2} d \mu_{\infty}
$$

where $Q_{\infty}$ denotes the $Q$-curvature with respect to $\left(J, \theta_{\infty}\right), \theta_{\infty}=e^{2 \lambda_{\infty}} \theta_{0}$, and $d \mu_{\infty}$ $=e^{4 \lambda_{\infty}} d \mu_{0}$. It follows that $Q_{\infty}=0$.

In the following, we are going to prove the smooth convergence for all time. First we want to prove that $\lambda$ converges to $\lambda_{\infty}$ in $L^{2}$. Write $\lambda_{\infty}=\lambda_{\infty}^{\perp}+\left(\lambda_{\infty}\right)_{\mathrm{ker}}$. Observe that $\left(\|\cdot\|_{2}\right.$ denotes the $L^{2}$ norm with respect to the volume form $\left.d \mu_{0}\right)$

$$
\begin{aligned}
\left\|\lambda_{\infty}^{\perp}-\lambda^{\perp}\right\|_{2} & \leq\left\|\lambda_{\infty}^{\perp}-\lambda^{\perp}\right\|_{S^{4,2}} \\
& \leq C\left\|2 P_{0}\left(\lambda_{\infty}^{\perp}-\lambda^{\perp}\right)\right\|_{2} \\
& =C\left\|2 P_{0} \lambda^{\perp}+Q_{0}^{\perp}\right\|_{2}
\end{aligned}
$$

by the subellipticity of $P_{0}$ on $\left(\operatorname{Ker} P_{0}\right)^{\perp}$ and $0=Q_{\infty}=e^{-4 \lambda_{\infty}}\left(2 P_{0} \lambda_{\infty}^{\perp}+Q_{0}^{\perp}\right)\left(\left(Q_{0}\right)_{\mathrm{ker}}\right.$ $=0$ by assumption). We compute

$$
\begin{aligned}
& \left|\mathcal{E}\left(\lambda_{\infty}^{\perp}\right)-\mathcal{E}\left(\lambda^{\perp}\right)\right| \\
& =\left|\int_{0}^{1} \frac{d}{d s} \mathcal{E}\left(\lambda^{\perp}+s\left(\lambda_{\infty}^{\perp}-\lambda^{\perp}\right)\right) d s\right| \\
& =\left|\int_{0}^{1} \int_{M}\left[2 P_{0}\left(\lambda^{\perp}+s\left(\lambda_{\infty}^{\perp}-\lambda^{\perp}\right)\right)+Q_{0}^{\perp}\right] \cdot\left(\lambda_{\infty}^{\perp}-\lambda^{\perp}\right) d \mu_{0} d s\right| \\
& \leq \int_{0}^{1}\left\|2 P_{0}\left(\lambda^{\perp}+s\left(\lambda_{\infty}^{\perp}-\lambda^{\perp}\right)\right)+Q_{0}^{\perp}\right\|_{2}|| \lambda_{\infty}^{\perp}-\lambda^{\perp} \|_{2} d s \\
& \leq C_{1}\left\|2 P_{0} \lambda^{\perp}+Q_{0}^{\perp}\right\|_{2}^{2}
\end{aligned}
$$

by the Cauchy inequality and (4.26). Let $\vartheta$ be a number between 0 and $\frac{1}{2}$. It follows from (4.27) that

$$
\left|\mathcal{E}\left(\lambda_{\infty}^{\perp}\right)-\mathcal{E}\left(\lambda^{\perp}\right)\right|^{1-\vartheta} \leq C_{2}|| 2 P_{0} \lambda^{\perp}+Q_{0}^{\perp}\left\|_{2}^{2(1-\vartheta)} \leq C_{2}\right\| 2 P_{0} \lambda^{\perp}+Q_{0}^{\perp} \|_{2}
$$


for $t$ large by noting that $2(1-\vartheta)>1$ and $\left\|2 P_{0} \lambda^{\perp}+Q_{0}^{\perp}\right\|_{2}$ tends to 0 as $t \rightarrow \infty$ by (4.25). Next we compute

$$
\begin{aligned}
& -\frac{d}{d t}\left(\mathcal{E}\left(\lambda^{\perp}\right)-\mathcal{E}\left(\lambda_{\infty}^{\perp}\right)\right)^{\vartheta} \\
& =-\vartheta\left(\mathcal{E}\left(\lambda^{\perp}\right)-\mathcal{E}\left(\lambda_{\infty}^{\perp}\right)\right)^{\vartheta-1} \frac{d}{d t}\left(\mathcal{E}\left(\lambda^{\perp}\right)-\mathcal{E}\left(\lambda_{\infty}^{\perp}\right)\right) \\
& =+\vartheta\left(\mathcal{E}\left(\lambda^{\perp}\right)-\mathcal{E}\left(\lambda_{\infty}^{\perp}\right)\right)^{\vartheta-1}\left\|2 P_{0} \lambda^{\perp}+Q_{0}^{\perp}\right\|_{2}\left\|\dot{\lambda}^{\perp}\right\|_{2} \\
& \geq \vartheta C_{2}^{-1}\left\|\dot{\lambda}^{\perp}\right\|_{2}
\end{aligned}
$$

by (1.7), (4.28), and noting that $\dot{\lambda}^{\perp}=-\left(2 P_{0} \lambda^{\perp}+Q_{0}^{\perp}\right)$ (see (3.10) $)$ and hence the left side is nonnegative. We learned the above trick of raising the power to $\vartheta$ from $[\underline{S}]$. Integrating (4.29) with respect to $t$ gives

$$
\int_{0}^{\infty}\left\|\dot{\lambda}^{\perp}\right\|_{2} d t<+\infty .
$$

Observing that $\frac{d}{d t}\left(\lambda^{\perp}-\lambda_{\infty}^{\perp}\right)=\dot{\lambda}^{\perp}$ and $-\frac{d}{d t}\left\|\lambda^{\perp}-\lambda_{\infty}^{\perp}\right\|_{2}^{2} \leq C\left\|\dot{\lambda}^{\perp}\right\|_{2}$, we can then deduce

$$
\lim _{t \rightarrow \infty}\left\|\lambda^{\perp}-\lambda_{\infty}^{\perp}\right\|_{2}^{2}=0
$$

by (4.30). On the other hand, we can estimate $|r(t)| \leq C\left\|2 P_{0} \lambda^{\perp}+Q_{0}^{\perp}\right\|_{2}=C\left\|\dot{\lambda}^{\perp}\right\|_{2}$ by (4.11). It follows from (4.30) that

$$
\int_{0}^{\infty}|r(t)| d t<+\infty .
$$

So in view of (4.8) and (4.32), $\lambda_{\text {ker }}$ converges to $\left(\lambda_{\infty}\right)_{\text {ker }}=\left(\lambda_{\text {ker }}\right)_{\infty}$ as $t \rightarrow \infty$ (not just a sequence of times). Since $\lambda_{\text {ker }}-\left(\lambda_{\infty}\right)_{\text {ker }}=-\int_{t}^{\infty} r(t) d t$ is a function of time only, we also have

$$
\lim _{t \rightarrow \infty}\left\|\lambda_{\mathrm{ker}}-\left(\lambda_{\infty}\right)_{\mathrm{ker}}\right\|_{S^{k, 2}}=0
$$

for any nonnegative integer $k$. With $\lambda^{\perp}$ replaced by $\lambda^{\perp}-\lambda_{\infty}^{\perp}$ in the argument to deduce (3.19), we obtain

$$
\frac{d}{d t} \int_{M}\left(\Delta_{0}^{k}\left(\lambda^{\perp}-\lambda_{\infty}^{\perp}\right)\right)^{2} d \mu_{0} \leq-4 \Upsilon \int_{M}\left(\Delta_{0}^{k}\left(\lambda^{\perp}-\lambda_{\infty}^{\perp}\right)\right)^{2} d \mu_{0}+C(k)\left\|\lambda^{\perp}-\lambda_{\infty}^{\perp}\right\|_{2}
$$


By Lemma 3.5 and (4.31), we get

$$
\lim _{t \rightarrow \infty} \int_{M}\left(\Delta_{0}^{k}\left(\lambda^{\perp}-\lambda_{\infty}^{\perp}\right)\right)^{2} d \mu_{0}=0
$$

Hence by the subellipticity of $\Delta_{0}^{k}$, we conclude from (4.35) and (4.31) that

$$
\lim _{t \rightarrow \infty}\left\|\lambda^{\perp}-\lambda_{\infty}^{\perp}\right\|_{S^{2 k, 2}}=0
$$

Together with 4.33, we have proved that $\lambda$ converges to $\lambda_{\infty}$ smoothly as $t \rightarrow \infty$.

\section{Essential Positivity of the $C R$ Paneitz Operator}

Let $(M, J, \theta)$ be a closed pseudohermitian 3-manifold with zero torsion. We will prove the essential positivity of the CR Paneitz operator $P$.

Since the torsion of $(M, J, \theta)$ is zero, the Lie derivative of the $C R$ structure $J$ with respect to $T$ (characteristic vector field) is zero. Hence $M$ admits a smooth $C R$ action of $R$, which is transverse to the contact bundle. This implies that $(M, J)$ is embeddable by Theorem 2.1. in [Le]. Therefore the $L^{2}$-closure of the Kohn Laplacian $\square_{b}$ has the closed range $([\mathrm{K} 1])$ and hence subelliptic estimates on the orthogonal complement of the kernel of $\square_{b}$ in $L^{2}$ ([K1], [K2]). We use the same symbol $\square_{b}$ to denote the $L^{2}$-closure.

On the other hand, since $\square_{b}$ is a closed operator, $\operatorname{Ker}\left(\square_{b}\right)$ is closed, and we have the following direct sum decomposition

$$
L^{2}=\operatorname{Ker}\left(\square_{b}\right) \oplus R\left(\square_{b}\right),
$$

where $R\left(\square_{b}\right)$ is the range of $\square_{b}$.

Let $C^{\infty}(M, C)$ be the space of all $C^{\infty}$ smooth complex-valued functions on $M$ and $C_{K}^{\infty}=C^{\infty}(M, C) \cap \operatorname{Ker}\left(\square_{b}\right)$. Then we have $C^{\infty}(M, C)=C_{K}^{\infty} \oplus\left(C_{K}^{\infty}\right)^{\perp}$. By (5.1) and subelliptic estimates on the orthogonal complement of the kernel of $\square_{b}$ in $L^{2}$, we see that $\left(C_{K}^{\infty}\right)^{\perp}=\left\{\square_{b} \varphi \mid \varphi \in C^{\infty}(M, C)\right\}$.

Let $H: C^{\infty}(M, C) \rightarrow C_{K}^{\infty}$ be the projection. Define the Green operator $G$ : $C^{\infty}(M, C) \rightarrow\left(C_{K}^{\infty}\right)^{\perp}$ by

$$
G(\alpha)=\omega \in\left(C_{K}^{\infty}\right)^{\perp}
$$


where $\omega$ is the unique solution of $\square_{b} \omega=\alpha-H(\alpha)$. It is easy to check that $G$ is symmetric and positive on $\left(C_{K}^{\infty}\right)^{\perp}$. Moreover, since $\square_{b}$ has subelliptic estimates, $G$ is compact on the orthogonal complement of $\operatorname{Ker}\left(\square_{b}\right)$.

Lemma 5.1. Let $\eta=\sup \left\{\|G \varphi\|: \varphi \in C^{\infty}(M, C),\|\varphi\|=1, \varphi \in\left(C_{K}^{\infty}\right)^{\perp}\right\}$. Then $\frac{1}{\eta}$ is an eigenvalue of $\square_{b}$.

Proof. Let $\left\{\varphi_{j}\right\} \subset\left(C_{K}^{\infty}\right)^{\perp}$ be a maximizing sequence for $\eta$, that is, $\left\|\varphi_{j}\right\|=1$ and $\left\|G \varphi_{j}\right\| \rightarrow \eta$. Since $G$ is symmetric, we have

$$
\begin{aligned}
\left\|G^{2} \varphi_{j}-\eta^{2} \varphi_{j}\right\|^{2} & =\left\|G^{2} \varphi_{j}\right\|^{2}-2 \eta^{2}\left\langle G^{2} \varphi_{j}, \varphi_{j}\right\rangle+\eta^{4} \\
& \leq \eta^{2}\left\|G \varphi_{j}\right\|^{2}-2 \eta^{2}\left\|G \varphi_{j}\right\|^{2}+\eta^{4}
\end{aligned}
$$

This inequality means that $\left\|G^{2} \varphi_{j}-\eta^{2} \varphi_{j}\right\| \rightarrow 0$ as $j \rightarrow 0$.

Let $\psi_{j}=G \varphi_{j}-\eta \varphi_{j}$. Since $G$ is positive on $\left(C_{K}^{\infty}\right)^{\perp}$, we have

$$
\begin{aligned}
\left\langle\psi_{j}, G^{2} \varphi_{j}-\eta^{2} \varphi_{j}\right\rangle & =\left\langle\psi_{j}, G \psi_{j}+\eta \psi_{j}\right\rangle \\
& =\left\langle\psi_{j}, G \psi_{j}\right\rangle+\eta\left\|\psi_{j}\right\|^{2} \\
& \geq \eta\left\|\psi_{j}\right\|^{2} .
\end{aligned}
$$

This inequality, together with the above, implies that $\left\|G \varphi_{j}-\eta \varphi_{j}\right\| \rightarrow 0$.

On the other hand, since $G$ is compact on the orthogonal complement of the kernel of $\square_{b}$, there exists a subsequence of $\left\{\varphi_{j}\right\}$, also denoted by $\left\{\varphi_{j}\right\}$, such that $\left\{G \varphi_{j}\right\}$ converges to a function $f \in L^{2}$. We have, for all $\varphi \in C^{\infty}(M, C)$,

$$
\begin{aligned}
\left\langle\left(\square_{b}-\frac{1}{\eta}\right) f, \varphi\right\rangle & =\left\langle f,\left(\square_{b}-\frac{1}{\eta}\right) \varphi\right\rangle \\
& =\lim _{j \rightarrow 0}\left\langle G \varphi_{j},\left(\square_{b}-\frac{1}{\eta}\right) \varphi\right\rangle \\
& =\lim _{j \rightarrow 0}\left\langle\varphi_{j}-\frac{1}{\eta} G \varphi_{j}, \varphi\right\rangle \\
& =\lim _{j \rightarrow 0} \frac{1}{\eta}\left\langle\eta \varphi_{j}-G \varphi_{j}, \varphi\right\rangle \\
& =0 .
\end{aligned}
$$

That is, $f \in L^{2}$ is a nontrivial weak solution of $\left(\square_{b}-\frac{1}{\eta}\right) f=0$. Actually $f \perp K e r \square_{b}$, so $f$ is smooth and $\frac{1}{\eta}$ is an eigenvalue of $\square_{b}$.

Lemma 5.2. Let $(M, J, \theta)$ be a closed pseudohermitian 3-manifold. Suppose that $(M, J)$ is embeddable. Then $\square_{b}$ has discrete eigenvalues: $0=\mu_{0}<\mu_{1} \leq \mu_{2} \leq$ 
$\cdots \leq \mu_{n} \rightarrow \infty$ and the corresponding eigenfunctions $\left\{\varphi_{i} \mid i \geq 1\right\}$ satisfying $\square_{b} \varphi_{i}=$ $\mu_{i} \varphi_{i}, \varphi_{i} \in C^{\infty}(M, C)$ can be chosen so that $\left\{\varphi_{i}\right\}$ forms an orthonormal basis of $\left(\operatorname{Ker} \square_{b}\right)^{\perp}$ in $L^{2}$.

Proof. Let $E(\mu)$ be the eigenspace with respect to the eigenvalue $\mu$. By the subelliptic estimates, $\operatorname{dim} E(\mu)<\infty$ if $\mu>0$.

For $j \geq 1$, let $\eta_{j} \equiv \sup \left\{\|G \varphi\|: \varphi \in C^{\infty}(M, C),\|\varphi\|=1, \varphi \in\left(\oplus_{i=0}^{j-1} E\left(\frac{1}{\eta_{i}}\right)\right)^{\perp}\right\}$. Here we denote $E\left(\frac{1}{\eta_{0}}\right)=C_{K}^{\infty}$. A similar argument as in Lemma 5.1 shows that $\frac{1}{\eta_{j}}$ is an eigenvalue of $\square_{b}$. Therefore we have the discrete eigenvalues $0=\mu_{0}<\mu_{1} \leq \mu_{2} \leq$ $\cdots \leq \mu_{n}, n \rightarrow \infty$. By the subelliptic estimates, $\left\{\mu_{n}\right\}$ can not have a finite limit. Thus $\mu_{n} \rightarrow \infty$ as $n \rightarrow \infty$.

Let $0=\mu_{0}<\mu_{1} \leq \mu_{2} \leq \cdots$ be the eigenvalues of $\square_{b}$, where each eigenvalue is included as many times as the dimension of its eigenspace, with a corresponding orthonormal sequence of eigenfunctions $\left\{\varphi_{i}\right\}$. Let $\alpha \in C^{\infty}(M, C)$, there exists $\beta \in$ $\left(C_{K}^{\infty}\right)^{\perp}$ such that $G \beta=\alpha-H(\alpha)$. It is easy to check that $\left\langle\alpha, \varphi_{i}\right\rangle \varphi_{i}=G\left(\left\langle\beta, \varphi_{i}\right\rangle \varphi_{i}\right)$, so we have

$$
\begin{aligned}
\left\|\alpha-\sum_{i=1}^{n}\left\langle\alpha, \varphi_{i}\right\rangle \varphi_{i}-H(\alpha)\right\| & =\left\|G \beta-\sum_{i=1}^{n}\left\langle\alpha, \varphi_{i}\right\rangle \varphi_{i}\right\| \\
& =\left\|G \beta-G\left(\sum_{i=1}^{n}\left\langle\beta, \varphi_{i}\right\rangle \varphi_{i}\right)\right\| \\
& =\left\|G\left(\beta-\sum_{i=1}^{n}\left\langle\beta, \varphi_{i}\right\rangle \varphi_{i}\right)\right\| \\
& \leq \frac{1}{\mu_{n+1}}\left\|\beta-\sum_{i=1}^{n}\left\langle\beta, \varphi_{i}\right\rangle \varphi_{i}\right\| \\
& \leq \frac{1}{\mu_{n+1}}\|\beta\| .
\end{aligned}
$$

Then $\mu_{n} \rightarrow \infty$ implies that

$$
\lim _{n \rightarrow \infty}\left\|\alpha-\sum_{i=1}^{n}\left\langle\alpha, \varphi_{i}\right\rangle \varphi_{i}-H(\alpha)\right\|=0 .
$$

Theorem 5.3. If the torsion of $(M, J, \theta)$ is zero, then the $C R$ Paneitz operator $P$ is essentially positive.

Proof. First, zero torsion implies that (i) the $C R$ Paneitz operator $P=\square_{b} \overline{\square_{b}}$, and (ii) the Kohn Laplacian $\square_{b}$ and $\bar{\square}_{b}$ commute, so they are diagonalized simultaneously on the finite dimensional eigenspace of $\square_{b}$ with respect to any nonzero eigenvalue. 
Therefore, we can choose an orthonormal basis $\left\{\varphi_{i}\right\}$ such that each eigenfunction $\in\left\{\varphi_{i}\right\}$ is also an eigenfunction of $\overline{\square_{b}}$, and hence, of $P$. We know that the eigenvalues of $\square_{b}$ (and hence of $\overline{\square_{b}}$ ) are all nonnegative. Therefore by Lemma 5.2, $P$ is essentially positive.

\section{Appendix A.}

We will give a brief introduction to pseudohermitian geometry (see [L1], [L2] for more details). Let $M$ be a closed 3-manifold with an oriented contact structure $\xi$. There always exists a global contact form $\theta$, obtained by patching together local ones with a partition of unity. The characteristic vector field of $\theta$ is the unique vector field $T$ such that $\theta(T)=1$ and $\mathcal{L}_{T} \theta=0$ or $d \theta(T, \cdot)=0$. A $C R$ structure compatible with $\xi$ is a smooth endomorphism $J: \xi \rightarrow \xi$ such that $J^{2}=-i d e n t i t y$. A pseudohermitian structure compatible with $\xi$ is a $C R$-structure $J$ compatible with $\xi$ together with a global contact form $\theta$.

Given a pseudohermitian structure $(J, \theta)$, we can choose a complex vector field $Z_{1}$, an eigenvector of $J$ with eigenvalue $i$, and a complex 1 -form $\theta^{1}$ such that $\left\{\theta, \theta^{1}, \theta^{\overline{1}}\right\}$ is dual to $\left\{T, Z_{1}, Z_{\overline{1}}\right\}$. It follows that $d \theta=i h_{1 \overline{1}} \theta^{1} \wedge \theta^{\overline{1}}$ for some nonzero real function $h_{1 \overline{1}}$. If $h_{1 \overline{1}}$ is positive, we call such a pseudohermitian structure $(J, \theta)$ positive, and we can choose a $Z_{1}$ (hence $\theta^{1}$ ) such that $h_{1 \overline{1}}=1$. That is to say

$$
d \theta=i \theta^{1} \wedge \theta^{\overline{1}}
$$

We will always assume our pseudohermitian structure $(J, \theta)$ is positive and $h_{1 \overline{1}}=1$ throughout the paper. The pseudohermitian connection of $(J, \theta)$ is the connection $\nabla^{\psi \cdot h .}$ on $T M \otimes C$ (and extended to tensors) given by

$$
\nabla^{\psi \cdot h \cdot} Z_{1}=\omega_{1}^{1} \otimes Z_{1}, \nabla^{\psi \cdot h \cdot} Z_{\overline{1}}=\omega_{\overline{1}}^{\overline{1}} \otimes Z_{\overline{1}}, \nabla^{\psi \cdot h \cdot} \cdot T=0
$$

in which the 1-form $\omega_{1}^{1}$ is uniquely determined by the following equation with a normalization condition:

$$
\begin{aligned}
d \theta^{1} & =\theta^{1} \wedge \omega_{1}{ }^{1}+A^{1}{ }_{\overline{1}} \theta \wedge \theta^{\overline{1}} \\
0 & =\omega_{1}{ }^{1}+\omega_{\overline{1}}{ }^{\overline{1}} .
\end{aligned}
$$


The coefficient $A^{1} \overline{1}_{1}$ in (A.1) is called the (pseudohermitian) torsion. Since $h_{1 \overline{1}}=1$, $A_{\overline{1} \overline{1}}=h_{1 \overline{1}} A^{1}{ }_{\overline{1}}=A^{1}{ }_{\overline{1}}$. And $A_{11}$ is just the complex conjugate of $A_{\overline{1} \overline{1}}$. Differentiating $\omega_{1}^{1}$ gives

$$
d \omega_{1}^{1}=W \theta^{1} \wedge \theta^{\overline{1}}+2 i \operatorname{Im}\left(A_{11, \overline{1}} \theta^{1} \wedge \theta\right)
$$

where $W$ is the Tanaka-Webster curvature.

We can define the covariant differentiations with respect to the pseudohermitian connection. For instance, $f_{1}=Z_{1} f, f_{1 \overline{1}}=Z_{\overline{1}} Z_{1} f-\omega_{1}^{1}\left(Z_{\overline{1}}\right) Z_{1} f$ for a (smooth) function $f$. We define the sub-gradient operator $\nabla_{b}$ and the sub-Laplacian operator $\Delta_{b}$ by

$$
\begin{aligned}
\nabla_{b} f & =f_{\overline{1}} Z_{1}+f_{1} Z_{\overline{1}}, \\
\Delta_{b} f & =f_{1 \overline{1}}+f_{\overline{1} 1} .
\end{aligned}
$$

respectively. We also define the Levi form $<,>_{J, \theta}$ by

$$
<V, U>_{J, \theta}=2 d \theta(V, J U)=v_{1} u_{\overline{1}}+v_{\overline{1}} u_{1}
$$

for $V=v_{1} Z_{\overline{1}}+v_{\overline{1}} Z_{1}, U=u_{1} Z_{\overline{1}}+u_{\overline{1}} Z_{1}$ in $\xi$ and

$$
(V, U)_{J, \theta}=\int_{M}<V, U>_{J, \theta} \theta \wedge d \theta
$$

For a vector $X \in \xi$, we define $|X|^{2} \equiv<X, X>_{J, \theta}$. It follows that $\left|\nabla_{b} f\right|^{2}=2 f_{1} f_{\overline{1}}$ for a real valued smooth function $f$. Also the square modulus of the sub-Hessian $\nabla_{b}^{2} f$ of $f$ reads $\left|\nabla_{b}^{2} f\right|^{2}=2 f_{11} f_{\overline{1} \overline{1}}+2 f_{1 \overline{1}} f_{\overline{1} 1}$. We recall below what the Folland-Stein space $S^{k, p}$ is. Let $D$ denote a differential operator acting on functions. We say $D$ has weight $m$, denoted $w(D)=m$, if $m$ is the smallest integer such that $D$ can be locally expressed as a polynomial of degree $m$ in vector fields tangent to the contact bundle $\xi$. We define the Folland-Stein space $S^{k, p}$ of functions on $M$ by

$$
S^{k, p}=\left\{f \in L^{p}: D f \in L^{p} \text { whenever } w(D) \leq k\right\}
$$

We define the $L^{p}$ norm of $\nabla_{b} f, \nabla_{b}^{2} f, \ldots$ to be $\left(\int\left|\nabla_{b} f\right|^{p} \theta \wedge d \theta\right)^{1 / p},\left(\int\left|\nabla_{b}^{2} f\right|^{p} \theta \wedge d \theta\right)^{1 / p}$, $\ldots$, respectively, as usual. So it is natural to define the $S^{k, p}$ norm of $f \in S^{k, p}$ as follows:

$$
\|f\|_{S^{k, p}} \equiv\left(\sum_{0 \leq j \leq k}\left\|\nabla_{b}^{j} f\right\|_{L^{p}}^{p}\right)^{1 / p}
$$


The function space $S^{k, p}$ with the above norm is a Banach space for $k \geq 0,1<p<\infty$.

There are also embedding theorems of Sobolev type. For instance, $S^{2,2} \subset S^{1,4}$ (for $\operatorname{dim} M=3$ ). We refer the reader to, for instance, [FS2] and [Fo] for more discussions on these spaces.

\section{REFERENCES}

[A] T. Aubin, Some Nonlinear Problems in Riemannian Geometry, Springer-Verlag, Berlin, 1998.

[B] S. Brendle, Global Existence and Convergence for a Higher-Order Flow in Conformal Geometry, Ann. Math., 158 (2003), 323-343.

[C] P. T. Chruściel, Semi-Global Existence and Convergence of solutions of the RobinsonTrautman (2-Dimensional Calabi) Equation, Commun. Math. Phys. 137 (1991), 289-313.

[C1] S.- C. Chang, The Q-Curvature Flow on a Closed 3-Manifold of Positive $Q$-Curvature, preprint.

[C2] S.- C. Chang, Recent Developments on the Calabi Flow, Contemporary Mathematics, 367, 17-42, A.M.S., 2005.

[C3] S.- C. Chang, Global Existence and Convergence of Solutions of the Calabi Flow on Riemann Surfaces of Genus $g \geq 2$, J. Math. Kyoto Univ. Vol. 40, No. 2 (2000), 363-377.

[C4] S.- C. Chang, The 2-dimensional Calabi Flow, Nagoya Math. J., to appear.

[CC1] S.- C. Chang and J.- H. Cheng, The Harnack Estimate for the Yamabe Flow on CR Manifolds of Dimension 3, Annals of Global Analysis and Geometry, 21 (2002), 111-121.

$[\mathrm{CH}]$ S.- S. Chern and R. Hamilton, On Riemannian Metrics Adapted to Three-dimensional Contact Manifolds, Lecture Notes in Math., 1111, 279-305, Springer-Verlag, 1984.

[Chi] H.-L. Chiu, The Sharp Lower Bound for the First Positive Eigenvalue of a Sub-Laplacian on a Three-Dimensional Pseudo-hermitian Manifold, submitted.

[CL] W. S. Cohn and G. Lu, Best Constants for Moser-Trudinger Inequalities on the Heisenberg Group, Indiana Univ. Math. J. 50 (2001), 1567-1591.

[CLe] J.- H. Cheng and J. M. Lee, The Burns-Epstein Invariant and Deformation of $C R$ Structures, Duke Math. J., 60 (1990), 221-254.

[CS] S.- C. Chen and M.- C. Shaw, Partial Differential Equations in several comlex variables, Studies in Advan. Math., 19, AMS/IP, 2001.

$[\mathrm{CW}]$ S.- C. Chang and C.- T. Wu, The Fourth-Order Q-Curvature Flow on Closed 3-Manifolds, Nagoya Math. J., to appear.

[Fa] F. Farris, An Intrinsic Construction of Fefferman's CR Metric, Pacific J. Math., 123 (1986), $33-45$. 
[FH] C. Fefferman and K. Hirachi, Ambient Metric Construction of $Q$-Curvature in Conformal and $C R$ Geometries, to appear in Math. Res. Lett..

[Fo] G. B. Folland, Subelliptic Estimates and Function Spaces on Nilpotent Lie Groups, Arkiv for Mat. 13 (1975), 161-207.

[FS1] G. B. Folland and E. M. Stein, Hardy spaces on homogeneous groups, Princeton U. Press, 1980.

[FS2] G. B. Folland and E. M. Stein, Estimates for the $\bar{\partial}_{b}$ Complex and Analysis on the Heisenberg Group, Comm. Pure Appl. Math., 27 (1974), 429-522.

[GG] A. R. Gover and C. R. Graham, $C R$ Invariant Powers of the Sub-Laplacian, preprint.

[GL] C. R. Graham and J. M. Lee, Smooth Solutions of Degenerate Laplacians on Strictly Pseudoconvex Domains, Duke Math. J., 57 (1988), 697-720.

[H] K. Hirachi, Scalar Pseudo-hermitian Invariants and the Szegö Kernel on 3-dimensional CR Manifolds, Lecture Notes in Pure and Appl. Math. 143, pp. 67-76, Dekker, 1992.

[JL] D. Jerison and J. M. Lee, The Yamabe Problem on CR Manifolds, J. Diff. Geom. 25 (1987), 167-197.

[K1] J. J. Kohn, Estimates for $\bar{\partial}_{b}$ on Compact Pseudoconvex $C R$ Manifolds, Proc. of Symposia in Pure Math., 43 (1985), 207-217.

[K2] J. J. Kohn, The Range of the Tangential Cauchy-Riemann Operator, Duke. Math. J., 53 (1986) 525-545.

[L1] J. M. Lee, Pseudo-Einstein Structure on CR Manifolds, Amer. J. Math. 110 (1988), 157-178.

[L2] J. M. Lee, The Fefferman Metric and Pseudohermitian Invariants, Trans. A.M.S. 296 (1986), 411-429.

[Le] L. Lempert, On Three-Dimensional Cauchy-Riemann Manifolds. J. of Amer. Math. Soc., 5 (1992), 923-969.

[P] S. Paneitz, A Quartic Conformally Covariant Differential Operator for Arbitrary PseudoRiemannian Manifolds, preprint, 1983.

[S] L. Simon, Asymptotics for a Class of Nonlinear Evolution Equations, with Applications to Geometric Problems, Ann. of Math., 118 (1983), 525-571.

[SC] Sánchez-Calle A., Fundamental Solutions and Geometry of the Sum of Squares of Vector Fields, Invent. Math., 78 (1984), 143-160. 
${ }^{1}$ Department of Mathematics, National Tsing Hua University, Hsinchu 30013, TaiWAN, R.O.C.

E-mail address: scchang@math.nthu.edu.tw

${ }^{2}$ Institute of Mathematics, Academia Sinica, Nankang, Taipei 11529, Taiwan, R.O.C. E-mail address: cheng@math.sinica.edu.tw

${ }^{3}$ Institute of Mathematics, Academia Sinica, Nankang, Taipei 11529, Tainan, R.O.C. E-mail address: hlchiu@math.sinica.edu.tw 